\title{
Peripheral sgp130-mediated trans-signaling blockade induces obesity and insulin resistance in mice via PPARa suppression
}

Tali Lanton ${ }^{1}$, Orr Levkovitch-Siany ${ }^{1}$, Shiran Udi ${ }^{3}$, Joseph Tam ${ }^{3}$, Rinat Abramovitch ${ }^{1,2}$, Sharon Perles $^{4}$, Evan Williams ${ }^{5}$, Jacob Rachmilewitz ${ }^{1}$, Uria Mor ${ }^{6}$, Eran Elinav ${ }^{6,7}$, Dirk Schmidt-Arras ${ }^{8}$, Ateequr Rehman ${ }^{9}$, Philip Rosenstiel ${ }^{9}$, Anastasios Giannou ${ }^{10}$, Samuel Huber ${ }^{10}$, Stefan Rose-John ${ }^{8}$, Eithan Galun $^{* 1,11}$, and Jonathan H. Axelrod ${ }^{* 1,11,12}$

Running Title: Elevated sgp130 induces obesity

${ }^{1}$ Goldyne Savad Institute of Gene Therapy, ${ }^{2}$ The Wohl Institute for Translational Medicine Hadassah Hebrew University Hospital, Jerusalem, Israel; ${ }^{3}$ Obesity and Metabolism Laboratory, Institute for Drug Research, School of Pharmacy, Faculty of Medicine, the Hebrew University of Jerusalem, Jerusalem, Israel; ${ }^{4}$ Raziel Therapeutics Ltd., Jerusalem, Israel; ${ }^{5}$ Institute of Molecular Systems Biology, ETH Zurich, Switzerland; ${ }^{6}$ Immunology Department, Weizmann Institute of Science, Rehovot, 7610001, ${ }^{7}$ Israel and Cancer-Microbiome Research Division, DKFZ, Heidelberg, 69120, Germany, ${ }^{8}$ Institute of Biochemistry and ${ }^{9}$ Institute of Clinical Molecular Biology, ChristianAlbrechts-University of Kiel, Kiel, Germany; ${ }^{10}$ University Medical Center Hamburg-Eppendorf, Hamburg, Germany; ${ }^{11}$ Senior Authors; ${ }^{12}$ Lead Contact.

*Correspondence: axelrod@hadassah.org.il; or EithanG@hadassah.org.il

Grant Support: This work was supported by grants to Jonathan H. Axelrod from the Israel Science Foundation (ISF 923/14), and the Israel Cancer Research Fund (8004906). The work of Eithan Galun (EG) was supported by grants from the NIH CA197081-02, the MOST, the ISF collaboration with Canada (2473/17), the personal ISF (486/17 and the ISF and ICORE - ISF (41/2011), the Deutsche Forschungsgemeinschaft, Bonn, Germany (SFB841). EG is also supported by the ERC Advance "RxmiRcanceR", and by the Kron, Raskin and the Robert Benson foundations. The work of Joseph Tam was supported by a grant from the Israel Science Foundation (ISF 1471/14). The work of Stefan Rose-John was supported by the Deutsche Forschungsgemeinschaft, Bonn, Germany (SFB877, Project A1; SFB841, Project C1) and by the Cluster of Excellence "Inflammation at Interfaces".

Disclosures- S.R.-J. is an inventor on patents owned by CONARIS Research Institute, which develops the sgp130Fc protein, and has stock ownership in CONARIS.

Author Contributions: EG and JHA conceived the study. EG, JHA, and TL designed the experiments. TL conducted the experiments. EG and JHA supervised the study. TL, OLS, RA, SU, EW, EE, HS, AG, and DS-A acquired data. TL, SU, JT, RA, SP, JR, SE, EE, AR, PR, DS-A, SRJ, 
EG, and JHA analyzed data. SRJ provided reagents. TL and JHA assembled the data and wrote the manuscript. All authors discussed the results and edited the manuscript.

Key Words: Interleukin-6 / Hepatosteatosis / Metabolic Syndrome / Obesity / sgp130 / trans-signaling

\section{Abstract}

IL-6 signaling via its receptor (IL-6R) and co-receptor (gp130) performs multiple roles in regulating metabolic homeostasis. However, gp130 is also expressed systemically in a soluble form (sgp130), which limits soluble IL-6 receptor (sIL-6R)-mediated signaling - also called trans-signaling. Here we find that transgenic peripheral sgp130-mediated trans-signaling blockade induces mature-onset obesity, while differentially affecting age-dependent behavioral determinants of energy expenditure. In youth, trans-signaling blockade increases feeding associated with reduced leptin sensitivity but increases energy expenditure to maintain metabolic balance. In aging, reduced physical activity predisposes mice to adiposity, adipose tissue macrophage recruitment, hepatosteatosis, hyperglycemia, and insulin resistance. Mechanistically, trans-signaling blockade reduces hepatic Stat3 phosphorylation and suppresses PPAR $\alpha$, associated with miR-21 upregulation, while pharmacological activation of PPAR $\alpha$ prevents obesity and hepatosteatosis, and rescues insulin sensitivity. Together these experiments reveal a role for peripheral IL-6 transsignaling in metabolic homeostasis and provide clinical significance to elevated sgp130 levels found in some obese and diabetic patients. 


\section{INTRODUCTION}

Substantial evidence indicates that IL-6 is crucial for maintaining metabolic homeostasis affecting multiple peripheral organs. Thus, mice with total IL-6 deficiency develop mature-onset obesity, characterized by increased adiposity and hepatic steatosis accompanied by glucose intolerance, hyperinsulinemia, and systemic insulin resistance (Matthews et al., 2010; Wallenius et al., 2002). Similarly, selective disruption in mice of IL- 6 signaling in the liver by targeted ablation of the IL-6 receptor (IL-6R) in hepatocytes increases inflammation and reduces insulin sensitivity (Wunderlich et al., 2010). Physical exercise has been found to induce elevation of muscle-derived IL-6 up to 100fold in the circulation (Pedersen \& Febbraio, 2008), and is associated in humans with the enhancement of insulin-stimulated glucose disposal and increased insulin sensitivity in the peripheral tissues (Carey et al., 2006). Moreover, long-term blockade of IL-6 signaling by ACTEMRA ${ }^{\circledR}$ (tocilizumab), a recombinant humanized anti-IL-6 receptor (IL-6R) antibody, is associated with significant increases in body weight and serum lipid and cholesterol levels and abolished the exercise-induced reduction in visceral adipose tissue mass (Kawashiri et al., 2011; Wedell-Neergaard et al., 2018).

Together with its systemic effects, IL-6 and its receptor are also constitutively expressed in the central nervous system (CNS) (Schobitz et al., 1993), where an increasing body of evidence points to its role in the regulation of metabolic homeostasis. IL-6-dependent pathways in the lateral parabrachial nucleus exerted control on body weight (Mishra et al., 2019) and in the hypothalamus, they contribute to reduce feeding and maintain peripheral glucose tolerance, especially in the face of leptin and insulin resistance in obesity (Timper et al., 2017). Thus, IL-6 interacts at both peripheral and central sites in the body, and these interactions, in general, appear to promote metabolic homeostasis (Jansson \& Palsdottir, 2015).

Notably, the cognate IL-6 receptor is found in two forms, a membrane-bound form (IL-6R) and an abundantly expressed soluble form (sIL-6R), leading to multiple signaling configurations. In its classical signaling configuration, membrane-bound IL-6R, which is expressed on limited cell populations, combines with IL- 6 to engage its ubiquitously expressed, transmembrane co-receptor, gp130, to initiate intracellular downstream signaling cascades, such as the JAK/STAT pathway (Heinrich et al., 2003). However, in its soluble-form, sIL-6R can form a complex with IL-6 and, in a mechanism called trans-signaling, initiate signaling in gp130 expressing cells, including in cells that do not express membrane-bound IL-6R (Rose-John et al., 2006). Importantly, gp130 is also produced in a truncated, soluble form (sgp130) that is normally expressed in the serum. There sgp130 functions to bind and neutralize IL-6/sIL-6R complexes, but not either protein alone, thus acting as a specific antagonist of trans-signaling (Jostock et al., 2001; Narazaki et al., 1993). It has been postulated that the abundant sgp 130 and sIL-6R in the serum act as a natural buffer to localize 
and limit the potentially deleterious effects of excessive IL-6 trans-signaling, such as found in some inflammation-driven pathologies (Rose-John et al., 2006). In contrast, in the hypothalamus, transsignaling underlies a crucial IL-6-mediated, leptin-independent mechanism that functions to suppress food-intake and maintain glucose tolerance especially in obesity (Timper et al., 2017).

However, the importance of trans-signaling in peripheral, metabolism-related IL-6 interactions remains unclear. In accordance with its role in immune activation, IL-6 trans-signaling is important for the obesity-associated recruitment of macrophages into adipose tissue in humans and also for macrophage chemotaxis in vitro (Kraakman et al., 2015), and as such would be expected to support glucose intolerance and insulin resistance in diabetes. Importantly, peripheral trans-signaling blockade can be imposed by high-level systemic expression of a recombinant sgp130 protein combined as a fusion protein with the fragment crystallizable $(\mathrm{Fc})$ domain of $\mathrm{IgG}$, called sgp $130 \mathrm{Fc}$ (Jostock et al., 2001), which has also been engineered into a transgenic mouse model (herein referred to as 'sgp130Fc mice') (Rabe et al., 2008). In this model, trans-signaling blockade in young, HFDchallenged mice prevents adipose tissue-associated macrophage (ATM) recruitment, but neither relieves nor exacerbates HFD-induced weight gain, hepatic steatosis, or insulin resistance in the young mice (Kraakman et al., 2015). Similarly, in young mice on a normal chow diet, ectopic sgp130 overexpression does not significantly alter body mass and does not affect glucose tolerance or energy expenditure in comparison to wild type controls (Kraakman et al., 2015). Paradoxically, however, elevated systemic sgp130 levels have been found in multiple studies to be associated with obesity, diabetes, and insulin resistance in adult patients (Nikolajuk et al., 2010; Weiss et al., 2013; Zuliani et al., 2010), although not in obese children or adolescents (De Filippo et al., 2015). Moreover, in patient populations with coronary artery disease (CAD) or at high risk for CAD, elevated serum levels of sgp130 were found in older individuals carrying the gp130 polymorphism, G148C, and were also associated with diabetes and increased body mass index (Wonnerth et al., 2014). This polymorphism has been shown to impair the functionality of gp130 (Benrick et al., 2008), thus suggesting that sgp130 polymorphisms may pose a potential genetic predisposition to co-morbidities of metabolic syndrome.

These observations suggest that peripheral trans-signaling may play an important role in the maintenance of metabolic homeostasis, but that aging is a critical factor in its presentation. In order to test this hypothesis and to unfold its potential mechanism(s), we raised sgp130Fc mice to the age of 14 months and assessed body composition, weight gain, blood glucose, and metabolic and behavioral determinants of energy expenditure in young versus aged mice. Our observations reveal that, in aging, blockade of peripheral trans-signaling induces striking behavioral and metabolic aberrations, including mature-onset obesity, glucose intolerance, and insulin resistance. We further identify the suppression of the Stat3-regulated miR-21/PPAR $\alpha$ axis as a mechanism underlying some aspects of this phenotype.

Page $\mid 4$ 


\section{RESULTS}

\section{Inhibition of trans-signaling induces mature-onset obesity on a normal chow diet}

Between the ages of about 5 and 6 months, the body weights of male sgp $130 \mathrm{Fc}$ mice begin to diverge from their WT littermates, becoming visibly and significantly overweight after 7 months of age (Figures 1A,B). The increase in body fat in mature sgp130Fc mice was shown by MRI imaging analysis (Figures 1C,D), by gonadal fat pad dissection upon sacrifice at 14 months (Figure 1E), and by echoMRI analysis (Figures $1 \mathrm{~F}$ and S1B). The increase in body weights was due largely to fat accumulation, which, according to MRI analysis, increased by about 40 percent in sgp130Fc mice at 14 months of age compared to their WT littermates, with roughly equal distribution between subcutaneous and abdominal fat mass (Figures 1D,E). Accordingly, prior to major bodyweight divergence the levels of the adipose-secreted hormone leptin were unchanged in young sgp $130 \mathrm{Fc}$ mice aged 2-7 months relative to controls, in agreement with previous observations (Kraakman et al., 2015), but increased significantly in comparison to their WT littermates by 12 months of age commensurate with their increased fat mass (Figure 2A).

\section{Etiology of weight gain in sgp130Fc mice}

To elucidate the underlying causes of body weight gain in sgp $130 \mathrm{Fc}$ mice we measured daily food intake and assessed energy expenditure using metabolic cages. Beginning from a young age (3 months) and prior to the onset of obesity, food intake by sgp130Fc mice was consistently increased by about 30 percent compared to WT littermates and remained high at older ages (Figure 2B). Interestingly, the elevated food intake levels, suggestive of leptin resistance, were consistent with the hyperleptinemia in older sgp130Fc mice, but were less clear for younger mice, which did not display increased serum leptin levels (Figure 2A). However, Western blot analysis following leptin injection revealed a strong reduction in leptin-induced Stat3 phosphorylation levels in the hypothalamus of young sgp130Fc mice (Figure 2C), thus indicating that prior to weight gain, leptinsensitivity is reduced in younger $\operatorname{sgp} 130 \mathrm{Fc}$ mice as well. This suggests that elevated peripheral trans-signaling blockade interferes with central leptin signaling, perhaps accounting in part for the increased food intake in the young sgp $130 \mathrm{Fc}$ mice, and later on in weight gain in the aged animals.

Metabolic cage analyses revealed that young (4 months) sgp130Fc mice also display overall increased physical activity, particularly voluntary nocturnal activity including wheel running, and elevated energy expenditure relative to WT littermates (Figures 2D and S1C). However, while nocturnal energy expenditure and voluntary physical activity decreased only marginally over time in WT controls, in sgp $130 \mathrm{Fc}$ mice both parameters decreased substantially such that by 13 months of age energy expenditure and wheel running were reduced, respectively, by 11 and 67 percent 
relative to their younger counterparts (Figures 2D, and S1C,D). Accordingly, sgp130Fc mice also displayed lower nocturnal respiratory quotient (RQ) that decreased further with age and elevated utilization of fat versus carbohydrate oxidation that decreased with age (Figure S1D). Statistical analyses confirmed the strong interaction between the metabolic phenotype of the sgp $130 \mathrm{Fc}$ mice and age $(P<0.001)$, indicating that age is likely a substantial factor in the mechanism of weight gain in the sgp130Fc mice (Figure 2D and S1D).

Importantly, the increased adiposity in sgp130Fc mice appeared to be due to adipocyte hypertrophy since, as assessed by H\&E staining of white adipose tissue (WAT) biopsies, a significant increase in the mean adipocyte cross-sectional area emerged concurrently with the onset of divergence in body weights in the mature mice (Figures 3A,B and S2A). In addition, ATM recruitment and crown-like structures characteristic of adipose tissue in obesity (Makki et al., 2013) increased in aged sgp $130 \mathrm{Fc}$ mice compared to controls, as quantified by anti-F4/80 immunostaining (Figures $3 \mathrm{C}, \mathrm{D}$ ) and confirmed by FACS analysis (Figure 3E). The increase in ATM in sgp130Fc mice also appears to be age-dependent since the opposite was reported for younger sgp $130 \mathrm{Fc}$ mice upon challenge with an HFD (Kraakman et al., 2015).

\section{Sgp130Fc mice display hyperglycemia, peripheral insulin resistance and hepatosteatosis in the absence of hepatic inflammation}

Because obesity and hyperglycemia are hallmarks of the metabolic syndrome, we next assessed the mice for glucose tolerance and insulin resistance at both early and late phases of weight gain. Sgp130Fc mice displayed fasting hyperglycemia (Figure 4A) and significant glucose intolerance that was exacerbated with age (Figure 4B and S3A). The overweight sgp $130 \mathrm{Fc}$ mice also displayed insulin tolerance test curves distinctive for peripheral insulin resistance (Figure 4C), and both fasting and post-prandial hyperinsulinemia compared to WT littermates (Figure 4D). Western blot analysis of ribosomal protein S6 phosphorylation in peripheral tissues (liver, muscle, and adipose) in response to injected insulin in 10-month old $\operatorname{sgp} 130 \mathrm{Fc}$ mice confirmed the presence of peripheral insulin resistance (Figure S3B-D). Thus, hyperglycemia in sgp130Fc mice is likely an outcome of peripheral insulin resistance consequent to the adiposity observed in the mature $\operatorname{sgp} 130 \mathrm{Fc}$ mice.

Since peripheral insulin resistance is also strongly associated with NAFLD and NASH (Gastaldelli, 2017), we determined whether long-term ectopic sgp $130 \mathrm{Fc}$ overexpression affected liver morphology and function by assessing lipid accumulation and inflammation in mature mice. Analysis of lipid accumulation by oil red O (ORO) staining showed that sgp130Fc livers at 14 months of age harbored frank steatosis with strikingly larger fat vacuoles compared to WT littermates, with initial signs of steatosis appearing in the mice already at 5 months of age (Figures 5A and S2B). These observations appear to indicate that elevated sgp130 recapitulates many phenotypic aspects previously reported for IL-6 knockout (IL-6 $\left.{ }^{-/}\right)$mice, including obesity, 
hyperglycemia and hepatic steatosis (Matthews et al., 2010; Wallenius et al., 2002). Curiously, however, in our hands, while IL- $6^{-/-}$mice displayed significant hepatic steatosis in comparison to littermates, they did so without obvious weight gain or hyperglycemia (Figure S4), suggesting that the effect of elevated sgp130 may be more complex than total IL-6 ablation.

A critical role of inflammatory signaling, associated in part with hepatic steatosis and increased Kupffer cells and mediated by tumor necrosis factor $\alpha(\mathrm{TNF} \alpha)$, has previously been identified in the induction of insulin resistance and glucose intolerance in mice with total IL-6 deficiency and also in mice with hepatocyte-targeted IL-6 receptor ablation (Matthews et al., 2010; Wunderlich et al., 2010). However, analysis of livers from aged (11-14 months old) sgp130Fc mice by flow cytometry, immunostaining, and qPCR revealed either reductions or no changes in the levels of monocytes/macrophages and inflammatory cytokine expression (Figures 5B and S5A). Thus, levels of $\mathrm{Cd} 11 \mathrm{~b}^{+}$monocytes in the liver assessed by flow cytometry were unchanged between sgp $130 \mathrm{Fc}$ and WT mice (Figure 5B), while populations of Kupffer cells and macrophages (F4/80) were either reduced (Figure 5B) or, as quantified by immunostaining, were unchanged (Figure S5B). Likewise, we observed no significant changes in levels of hepatic mRNAs encoding $C d 11 b, F 4 / 80, C d 68$, and Tlr4, or in the expression of inflammatory cytokines, Tnfa, Il-10 and Il-6 (Figure S5C). These observations suggest that in aged sgp130Fc mice hepatic inflammation-independent pathways may contribute to the development of insulin resistance and glucose intolerance.

\section{Suppression of hepatic PPARa signaling and miR-21 upregulation are associated with IL-6 trans-signaling blockade}

IL-6 has been found to increase expression of hepatic nuclear transcription factor peroxisome proliferator-activated receptor alpha (PPAR $\alpha$ ), which is a master regulator of lipid metabolism, and has been found to be decreased in humans and mice with fatty liver disease (Liss \& Finck, 2017; Montagner et al., 2016). Interestingly, Ppara expression can also be suppressed by miR-21, which targets PPAR $\alpha$ expression in fatty liver disease (Kida et al., 2011; Loyer et al., 2016). Since miR21 can be suppressed by signal transducer and activator of transcription 3 (Stat3) (Zhang et al., 2011), a primary mediator of IL-6 signal transduction, we hypothesized that suppression of PPAR $\alpha$ associated with the reduction of Stat 3 and elevation of miR-21 may be involved in the metabolic phenotype of our sgp130Fc mice.

Consistent with this hypothesis, we found that levels of phosphorylated Stat3 in livers of sgp $130 \mathrm{Fc}$ mice were significantly reduced compared to WT littermates (Figure 6A). Additionally, we found that expression of miR-21 was consistently enhanced in livers of sgp130Fc mice (Figure 6B), while levels of miR-122, a liver-specific micro-RNA previously implicated in fat metabolism (Chai et al., 2017; Esau et al., 2006), did not change in a consistent manner between sgp130Fc and WT mice 
(Figure 6B). Analysis of Ppara mRNA and protein levels in livers of sgp130Fc and WT littermates at 2, 5, or 14 months of age showed significant reductions of Ppar $\alpha$ mRNA expression in sgp $130 \mathrm{Fc}$ mice relative to controls at 2 and 14 months (Figure 6C) and of PPAR $\alpha$ protein as well, as shown at 5 months of age (Figure 6D). Importantly, compared to WT controls, trans-signaling blockade reduced mRNA levels of Ppar $\alpha$ regulated genes, including Cpt1, Apoe, and Fasn (Hong et al., 2004; Vida et al., 2013) (Figure 6C).

To confirm the role of phosphorylated Stat 3 in the regulation of PPAR $\alpha$, we examined the effect of hepatocyte-targeted Stat3 ablation in Alb-CreSTAT3 ${ }^{\text {floxP }}$ (Stat $3^{\text {shep }}$ ) mice (Inoue et al., 2004) on hepatic miR-21 and PPAR $\alpha$ expression levels. Consistent with previous reports, Stat $3^{\text {shep }}$ mice displayed notable obesity from 6 months of age, as well as fasting hyperglycemia that was observed at 12 months although not at 6 months of age (Figures S6A,B) in addition to notable hepatic steatosis at 12 months (Figures 6C). Importantly, the Stat $3^{\Delta \text { hep }}$ mice also displayed significantly elevated miR21 levels, but not miR-122, together with reduced expression of Ppar $\alpha$ mRNA and a marginal reduction in its downstream target gene, $C p t 1$ (Figure S6D).

\section{Activation of PPARa normalizes body weight, fat accumulation and glucose tolerance in mature sgp130Fc mice}

To determine whether suppression of PPAR $\alpha$ contributes to the metabolic phenotype of sgp $130 \mathrm{Fc}$ mice, we tested the ability of the PPAR $\alpha$ agonist, fenofibrate (Chaput et al., 2000), to rescue the sgp130Fc metabolic phenotype. We placed sgp130Fc mice and WT littermates on a normal chow diet with or without fenofibrate for 2.5 months, beginning at 4.5 months of age, prior to body weight divergence, and upon completion assessed body weight, fat accumulation, and serum glucose levels and insulin resistance (Figure 7A). Analysis of mRNA levels of PPAR $\alpha$ target genes in the livers of naïve and fenofibrate treated sgp $130 \mathrm{Fc}$ and WT mice confirmed the fenofibrate stimulated increase in PPAR $\alpha$ activity. Most of the evaluated PPAR $\alpha$ target genes in the liver, including Cyp4a10, Cyp4a14, Fabp3, Fatp, L-Fabp and Vnn1 (de la Rosa Rodriguez et al., 2018; Martin et al., 1997; Montagner et al., 2016; van Diepen et al., 2014; Vida et al., 2013), were strongly elevated by fenofibrate (Figure S7); but interestingly, did not have the same effect on Cpt-1 and Apoe (Figure S7). Importantly, fenofibrate treatment reduced the excess body weight (Figure 7B) and gonadal fat pad weight (Figure 7B,C) of sgp130Fc mice, bringing them to levels identical to those of fenofibrate treated WT mice, whose weight was also reduced by about 14 percent in comparison to untreated WT littermate controls. Fenofibrate treatment also reduced fasting glucose levels in the sgp130Fc mice to those of fenofibrate treated WT controls (Figure 7D), and rescued insulin sensitivity in the sgp130Fc mice (Figure 7E). Moreover, hepatic steatosis, which was varyingly present at 7 months of age in sgp $130 \mathrm{Fc}$ mice fed a normal chow diet was strikingly reduced to WT levels by fenofibrate 
treatment (Figure 7F). Statistical analyses confirmed the strong strain-dependent interaction of the response to the fenofibrate diet for multiple parameters, including body weight $(P=0.02)$, gonadal fat pad weight $(P=0.005)$, serum glucose levels $(P=0.008)$, and upregulation of $L-F a b p$ mRNA expression $(P=0.028)$ (Figures 7 and S7). Thus, fenofibrate treatment brought greater overall reductions in the metabolic imbalance of the sgp $130 \mathrm{Fc}$ mice relative to the correspondingly treated WT controls, indicating that reduced PPAR $\alpha$ levels likely constitutes part of the mechanism leading to weight gain in the sgp130Fc mice. Together, this strongly suggests that suppression of PPAR $\alpha$, perhaps mediated in part by miR-21 upregulation consequent to the trans-signaling blockadeinduced reduction in Stat3 phosphorylation, contributes substantially to the aberrant metabolic phenotype of spg $130 \mathrm{Fc}$ mice.

\section{DISCUSSION}

Co-morbidities of metabolic syndrome are an enormous healthcare burden for western societies and are becoming increasingly so for developing countries as well (Hruby \& Hu, 2015). Along with weight, genetics, lifestyle, and excess caloric intake, aging is considered one of the most important independent determining risk factors in the development of metabolic syndrome (Bonomini et al., 2015). Indeed, many of the conditions contributing to metabolic syndrome, such as obesity, insulin resistance, and inflammation, also increase in prevalence during aging (Bonomini et al., 2015). IL6 has long been seen as a pro-inflammatory cytokine, with functions that parallel those of TNF $\alpha$ as a driver of obesity-associated metabolic complications. However, ample evidence also indicates that IL-6 signaling is a crucial regulator of metabolic homeostasis, and, importantly, contributes centrally through a mechanism of trans-signaling to affect body weight control, reduce feeding, and maintain peripheral glucose tolerance, especially in obesity (Timper et al., 2017).

Our findings demonstrate that in aging peripheral trans-signaling also contributes to the control of behavioral determinants of energy expenditure and to maintain metabolic homeostasis. Thus, following trans-signaling blockade energy expenditure imbalance is manifest at an early age with hyperphagia balanced by hyperactivity and in aging with ectopic lipid accumulation, including hypertrophy-related adiposity and hepatic steatosis, glucose intolerance, and insulin resistance, together with reduced physical activity.

Blockade of peripheral trans-signaling in sgp130Fc mice recapitulates many of the metabolismrelated phenotypic aspects found in some strains of $\mathrm{IL}_{-} 6^{-/}$and also Stat $3^{\Delta \mathrm{Hep}}$ mice (Inoue et al., 2004; Matthews et al., 2010; Wallenius et al., 2002), but with striking and perhaps instructive differences. For instance, sgp130Fc mice display hyperphagia from an early age, while obese IL-61- mice and Stat $3^{\Delta \text { Hep }}$ mice do not. While the molecular and cellular mechanism(s) leading to 
hyperactivity and hyperphagia in sgp130Fc mice are not entirely clear, our observation that ectopic sgp130 expression reduces leptin signaling in the hypothalamus is in agreement with previous reports that IL-6 trans-signaling in the hypothalamus functions to limit food-intake especially in obesity (Timper et al., 2017). However, in our model, the sgp130Fc transgene is expressed peripherally under the PEPCK promoter, and the recombinant protein does not cross the blood-brain barrier (Braun et al., 2013; Rabe et al., 2008).

How peripheral trans-signaling blockade reduces leptin signaling in the hypothalamus is presently unclear. Various mechanisms have been shown to underlie leptin resistance in obesity in experimental models. These include reduced leptin passage across the blood-brain barrier into the CNS, which can also be inhibited by triglycerides and TNF- $\alpha$, impairment of leptin receptor (LepRb) trafficking, suppression of LepRb signaling, and also environmental effects such as neonatal overfeeding (Banks et al., 2004; Cui et al., 2017; Jung \& Kim, 2013). Thus, it is conceivable that trans-signaling blockade in the periphery may impede leptin signaling indirectly by affecting one of these pathways. Alternatively, sgp130Fc may act directly to impede leptin signaling. Further studies are required to elucidate the mechanism(s) through which peripheral trans-signaling contributes to mediate the cross-talk between the periphery and CNS regulated behavioral functions of appetite and activity.

The observation that young spg130Fc mice do not display weight gain despite their hyperphagia suggests that factors distinct from hyperphagia may also contribute to obesity and metabolic imbalance also in these mice. Glucose intolerance in IL-6 signaling-deficient mice challenged with an HFD has consistently been found to be associated with an exacerbated inflammatory response in the liver and adipose tissue, suggesting that IL-6 may have a unique homeostatic role in limiting the low-grade inflammatory state thought to be a critical driver in the development of glucose intolerance (Matthews et al., 2010; Mauer et al., 2014; Wunderlich et al., 2010). However, in our sgp130Fc mice, trans-signaling blockade induced glucose intolerance in the absence of hepatic inflammation. This observation suggests that trans-signaling mediated prevention of glucose intolerance and its role in metabolic homeostasis may operate via a mechanism(s) other than the limitation of low-grade hepatic inflammation.

Here we identify PPAR $\alpha$ suppression in the liver to be a critical mechanism in the onset of matureonset weight gain, hepatic steatosis, and insulin resistance in trans-signaling deficient mice, which may be mediated by reduced STAT3 suppression of miR-21 expression. This conclusion is consistent with previous reports showing PPAR $\alpha$ to be an important, miR-21-targeted regulator of metabolism, which is also reduced in human NAFLD patients (Liss \& Finck, 2017), and with reports demonstrating that miR-21 is suppressed by STAT3 (Zhang et al., 2011). However, considering that the phenotypes associated with trans-signaling blockade are broader in nature and appear earlier than those associated with hepatocyte-targeted STAT3 ablation alone, it is also clear that Page | 10 
suppression of STAT3 and PPAR $\alpha$ in hepatocytes can account only in part for the phenotype induced by trans-signaling blockade. Curiously, while PPAR $\alpha$ suppression in sgp130Fc mice was evident from an early age, the metabolic consequences of IL-6/STAT3 signaling-disruption described here and by others (Inoue et al., 2004; Matthews et al., 2010; Wallenius et al., 2002) are strongly age-dependent, appearing in mice only after about 6 months of age. This discrepancy suggests that critical factors may come into play in aging to allow the metabolic imbalance induced by trans-signaling blockade and PPAR $\alpha$ suppression. In this sense, the reduction in physical activity displayed by mature sgp $130 \mathrm{Fc}$ mice, which is reminiscent of the sedentary behavior displayed by some adult humans, may be of relevance; although, how trans-signaling interacts with aging-related factors to modify physical activity is presently unclear.

It is perhaps important to note that in addition to IL-6 trans-signaling, sgp130 has also been observed to partially inhibit signaling by other IL-6 family members in vitro, including, interleukin-11 leukemia inhibitory factor (LIF), ciliary neurotrophic factor, and oncostatin M (OSM), albeit with about 100-fold lower efficacy (Jostock et al., 2001; Narazaki et al., 1993). Thus, it is reasonable to expect that the metabolic phenotype observed in $\mathrm{sgp} 130 \mathrm{Fc}$ mice may be broader in scope than that observed in IL-6 knockout mice, and is consistent with the broad roles of IL-6 family cytokines in metabolic homeostasis (Pasquin et al., 2016).

Lastly, it is intriguing that although these aberrant metabolic traits appear common to many strains of mice that carry mutations affecting IL-6/STAT3 signaling, they reportedly are not shared by all strains of IL-6 ${ }^{-/}$and Stat $3^{\text {Ahep }}$ mice, despite carrying similar or identical mutant alleles (Di Gregorio et al., 2004; Moh et al., 2007). In line with this observation, our IL-6 ${ }^{-/-}$mice displayed mature-onset hepatic steatosis, but do not become notably obese. Similarly, the obese phenotype of aged sgp130Fc mice seen here has not been observed in other globally dispersed sgp $130 \mathrm{Fc}$ colonies. These include the sgp130Fc colonies located in Hamburg and Kiel, which did not show the herein described obese phenotype (SRJ, personal communication); although, a trend of increasing fat mass has been observed by others in young sgp130Fc mice (Kammoun et al., 2017). These observations suggest that subtle, and yet unidentified genetic, epigenetic, or perhaps environmental factors may influence the penetrance of metabolic phenotypes related to IL-6, STAT3, and trans-signaling deficiencies. By analyzing the microbiome of sgp130Fc and WT strains maintained in animal facilities in three different geographical locations (Jerusalem, Israel, and Kiel and Hamburg, Germany), we find major differences between the animal houses, which by PCoA analysis are highly statistically significant $(P=0.001)$ (Figure S8, Table SI). The microbiome in Jerusalem has a higher Clostridial population and fewer Bactericides. The literature perceives Bacteroides as a beneficial Genus and Clostridial as a harming Genus (Wexler, 2007; Woting et al., 2014). The metabolic effects we see in the present study, although being strain-dependent are possibly also affected by the differences in the microbiome seen in the different animal facilities. These Page $\mid 11$ 
observations warrant further investigations into the connection between specific bacterial strains and their metabolic effects.

Taken together, our findings demonstrate a role for peripheral IL-6 trans-signaling in metabolic homeostasis in aging and thus provide clinical significance to the apparent association of elevated sgp130 levels with obesity, insulin resistance, and diabetes found in some human patients (Nikolajuk et al., 2010; Weiss et al., 2013; Wonnerth et al., 2014; Zuliani et al., 2010). Future research is required to unravel the mechanism(s) through which peripheral trans-signaling affects leptin resistance, food-intake and activity, and to identify aging-related determinants that it appears to counter in maintaining metabolic homeostasis. Lastly, our experiments identify sgp130 and IL-6 trans-signaling affected pathways as potential drug targets for obesity and obesity-associated insulin and leptin resistance in aging.

\section{Materials and Methods}

\section{Animal Care}

Mice were maintained in an animal facility with a temperature of $\sim 23^{\circ} \mathrm{C}$ in a 12 -hour light-dark cycle, under SPF conditions and received sterile commercial rodent chow and water ad libitum. Maintenance of mice and all experimental procedures were performed in accordance with the Institutional Animal Care and Use Committee approved animal treatment protocols (license number OPRR-A01-5011).

\section{Genetic mouse models}

Male mice were used in all experiments. Sgp130Fc ${ }^{+/+}(\mathrm{C} 57 \mathrm{BL} / 6 \mathrm{~N})$ mice (Rabe et al., 2008) were crossed with WT (C57BL/6J) mice purchased from Harlan Laboratories (Jerusalem, Israel) to generate heterozygous sgp $130 \mathrm{Fc}^{+/-}$mice. $\mathrm{IL}_{-6^{+/-}}$mice were then crossed in order to generate both homozygous IL- $6^{-/-}$mice and wild type (WT) $\left(\mathrm{IL}^{-} 6^{+/+}\right)$littermates. Stat $3^{\text {flox/lox }}$ Alb-Cre/ $\left(\mathrm{Stat}^{3 \mathrm{Hep}}\right)$ mice were generated from STAT3 (Stat3 ${ }^{\text {flox/lox }}$ ) (C57BL/6) mice crossed with Alb-Cre ${ }^{+/+}$(C57BL/6) mice. See Supplementary Information for further details in the generation and validation of genetic mouse models.

\section{Study design}

Male mice were used in all experimental groups were housed in groups consisting of 2-3 mice per cage. Sgp130Fc mice and WT littermates were raised to the age of 14 months. Experimental cohorts were sacrificed at 2, 5, and 14 months of age. Stat $3^{\Delta \mathrm{Hep}}$ and Stat $3^{\text {flox }}$ littermates were grown to 12 months of age. Upon sacrifice of the cohorts, liver and adipose tissue samples were removed and snap-frozen in liquid nitrogen for protein and RNA extraction, or embedded for frozen tissue 
sections or fixed in 4 percent formaldehyde and paraffin-embedded for histological and immunostaining analyses. For full details analyses concerning MRI, metabolic cages, food intake, leptin resistance, glucose and insulin tolerance testing, insulin secretion, insulin signaling, Western blot, histochemical and immunostaining, RNA, flow cytometry, and taxonomic microbiota see Supplementary Information.

\section{Statistical Analysis}

Data were evaluated for significance by two-tailed Student's $t$-test or Mann-Whitney test, unless otherwise noted. $P \leq 0.05$ was considered significant for all analyses. Calculations were performed using GraphPad Prism 6.02 software (Graph-Pad Software, Inc, San Diego, CA) unless otherwise noted.

\section{Acknowledgments}

The authors are grateful to Deborah Olam for excellent technical assistance; to Catherine TempelBrami and Yael S. Schiffenbauer for assistance in performing MRI experiments; to Neta Barashi, Ann Reisch Saada for helpful discussions; and to Tali Bdolah-Abram for expert advice concerning statistical evaluations.

\section{References}

Banks, W. A., Coon, A. B., Robinson, S. M., Moinuddin, A., Shultz, J. M., Nakaoke, R., \& Morley, J. E. (2004). Triglycerides induce leptin resistance at the blood-brain barrier. Diabetes, 53(5), 1253-1260. doi:10.2337/diabetes.53.5.1253

Benrick, A., Jirholt, P., Wernstedt, I., Gustafsson, M., Scheller, J., Eriksson, A. L., Boren, J., Hedner, T., Ohlsson, C., Hard, T., Rose-John, S., \& Jansson, J. O. (2008). A nonconservative polymorphism in the IL-6 signal transducer (IL6ST)/gp130 is associated with myocardial infarction in a hypertensive population. Regul Pept, 146(1-3), 189-196. doi:10.1016/j.regpep.2007.09.031

Bonomini, F., Rodella, L. F., \& Rezzani, R. (2015). Metabolic syndrome, aging and involvement of oxidative stress. Aging Dis, 6(2), 109-120. doi:10.14336/AD.2014.0305

Braun, O., Dewitz, C., Moller-Hackbarth, K., Scheller, J., Schiffelholz, T., Baier, P. C., \& RoseJohn, S. (2013). Effects of blockade of peripheral interleukin-6 trans-signaling on hippocampus-dependent and independent memory in mice. J Interferon Cytokine Res, 33(5), 254-260. doi:10.1089/jir.2012.0096

Carey, A. L., Steinberg, G. R., Macaulay, S. L., Thomas, W. G., Holmes, A. G., Ramm, G., Prelovsek, O., Hohnen-Behrens, C., Watt, M. J., James, D. E., Kemp, B. E., Pedersen, B. K., \& Febbraio, M. A. (2006). Interleukin-6 increases insulin-stimulated glucose disposal in humans and glucose uptake and fatty acid oxidation in vitro via AMP-activated protein kinase. Diabetes, 55(10), 2688-2697. doi:10.2337/db05-1404 
Chai, C., Rivkin, M., Berkovits, L., Simerzin, A., Zorde-Khvalevsky, E., Rosenberg, N., Klein, S., Yaish, D., Durst, R., Shpitzen, S., Udi, S., Tam, J., Heeren, J., Worthmann, A., Schramm, C., Kluwe, J., Ravid, R., Hornstein, E., Giladi, H., \& Galun, E. (2017). Metabolic Circuit Involving Free Fatty Acids, microRNA 122, and Triglyceride Synthesis in Liver and Muscle Tissues. Gastroenterology, 153(5), 1404-1415. doi:10.1053/j.gastro.2017.08.013

Chaput, E., Saladin, R., Silvestre, M., \& Edgar, A. D. (2000). Fenofibrate and rosiglitazone lower serum triglycerides with opposing effects on body weight. Biochem Biophys Res Commun, 271(2), 445-450. doi:10.1006/bbrc.2000.2647

Cui, H., Lopez, M., \& Rahmouni, K. (2017). The cellular and molecular bases of leptin and ghrelin resistance in obesity. Nat Rev Endocrinol, 13(6), 338-351. doi:10.1038/nrendo.2016.222

De Filippo, G., Rendina, D., Moccia, F., Rocco, V., \& Campanozzi, A. (2015). Interleukin-6, soluble interleukin-6 receptor/interleukin-6 complex and insulin resistance in obese children and adolescents. J Endocrinol Invest, 38(3), 339-343. doi:10.1007/s40618-0140176-4

de la Rosa Rodriguez, M. A., Sugahara, G., Hooiveld, G., Ishida, Y., Tateno, C., \& Kersten, S. (2018). The whole transcriptome effects of the PPARalpha agonist fenofibrate on livers of hepatocyte humanized mice. BMC Genomics, 19(1), 443. doi:10.1186/s12864-018-4834-3

Di Gregorio, G. B., Hensley, L., Lu, T., Ranganathan, G., \& Kern, P. A. (2004). Lipid and carbohydrate metabolism in mice with a targeted mutation in the IL- 6 gene: absence of development of age-related obesity. Am J Physiol Endocrinol Metab, 287(1), E182-187. doi:10.1152/ajpendo.00189.2003

Esau, C., Davis, S., Murray, S. F., Yu, X. X., Pandey, S. K., Pear, M., Watts, L., Booten, S. L., Graham, M., McKay, R., Subramaniam, A., Propp, S., Lollo, B. A., Freier, S., Bennett, C. F., Bhanot, S., \& Monia, B. P. (2006). miR-122 regulation of lipid metabolism revealed by in vivo antisense targeting. Cell Metab, 3(2), 87-98. doi:10.1016/j.cmet.2006.01.005

Gastaldelli, A. (2017). Insulin resistance and reduced metabolic flexibility: cause or consequence of NAFLD? Clin Sci (Lond), 131(22), 2701-2704. doi:10.1042/CS20170987

Heinrich, P. C., Behrmann, I., Haan, S., Hermanns, H. M., Muller-Newen, G., \& Schaper, F. (2003). Principles of interleukin (IL)-6-type cytokine signalling and its regulation. Biochem J, 374(Pt 1), 1-20. doi:10.1042/BJ20030407 BJ20030407 [pii]

Hong, F., Radaeva, S., Pan, H. N., Tian, Z., Veech, R., \& Gao, B. (2004). Interleukin 6 alleviates hepatic steatosis and ischemia/reperfusion injury in mice with fatty liver disease. Hepatology, 40(4), 933-941.

Hruby, A., \& Hu, F. B. (2015). The Epidemiology of Obesity: A Big Picture. Pharmacoeconomics, 33(7), 673-689. doi:10.1007/s40273-014-0243-x

Inoue, H., Ogawa, W., Ozaki, M., Haga, S., Matsumoto, M., Furukawa, K., Hashimoto, N., Kido, Y., Mori, T., Sakaue, H., Teshigawara, K., Jin, S., Iguchi, H., Hiramatsu, R., LeRoith, D., Takeda, K., Akira, S., \& Kasuga, M. (2004). Role of STAT-3 in regulation of hepatic gluconeogenic genes and carbohydrate metabolism in vivo. Nat Med, 10(2), 168-174.

Jansson, J. O., \& Palsdottir, V. (2015). Brain IL-6--Where Amylin and GLP-1 Antiobesity Signaling Congregate. Diabetes, 64(5), 1498-1499. doi:10.2337/db14-1910

Jostock, T., Mullberg, J., Ozbek, S., Atreya, R., Blinn, G., Voltz, N., Fischer, M., Neurath, M. F., \& Rose-John, S. (2001). Soluble gp130 is the natural inhibitor of soluble interleukin-6 receptor transsignaling responses. Eur J Biochem, 268(1), 160-167.

Jung, C. H., \& Kim, M. S. (2013). Molecular mechanisms of central leptin resistance in obesity. Arch Pharm Res, 36(2), 201-207. doi:10.1007/s12272-013-0020-y

Kammoun, H. L., Allen, T. L., Henstridge, D. C., Kraakman, M. J., Peijs, L., Rose-John, S., \& Febbraio, M. A. (2017). Over-expressing the soluble gp130-Fc does not ameliorate methionine and choline deficient diet-induced non alcoholic steatohepatitis in mice. PLoS One, 12(6), e0179099. doi:10.1371/journal.pone.0179099

Kawashiri, S. Y., Kawakami, A., Yamasaki, S., Imazato, T., Iwamoto, N., Fujikawa, K., Aramaki, T., Tamai, M., Nakamura, H., Ida, H., Origuchi, T., Ueki, Y., \& Eguchi, K. (2011). Effects of the anti-interleukin- 6 receptor antibody, tocilizumab, on serum lipid levels in patients with rheumatoid arthritis. Rheumatol Int, 31(4), 451-456. doi:10.1007/s00296-009-1303-y 
Kida, K., Nakajima, M., Mohri, T., Oda, Y., Takagi, S., Fukami, T., \& Yokoi, T. (2011). PPARalpha is regulated by miR-21 and miR-27b in human liver. Pharm Res, 28(10), 2467-2476. doi:10.1007/s11095-011-0473-y

Kraakman, M. J., Kammoun, H. L., Allen, T. L., Deswaerte, V., Henstridge, D. C., Estevez, E., Matthews, V. B., Neill, B., White, D. A., Murphy, A. J., Peijs, L., Yang, C., Risis, S., Bruce, C. R., Du, X. J., Bobik, A., Lee-Young, R. S., Kingwell, B. A., Vasanthakumar, A., Shi, W., Kallies, A., Lancaster, G. I., Rose-John, S., \& Febbraio, M. A. (2015). Blocking IL-6 trans-signaling prevents high-fat diet-induced adipose tissue macrophage recruitment but does not improve insulin resistance. Cell Metab, 21(3), 403-416. doi:10.1016/j.cmet.2015.02.006

Liss, K. H., \& Finck, B. N. (2017). PPARs and nonalcoholic fatty liver disease. Biochimie, 136, 6574. doi:10.1016/j.biochi.2016.11.009

Loyer, X., Paradis, V., Henique, C., Vion, A. C., Colnot, N., Guerin, C. L., Devue, C., On, S., Scetbun, J., Romain, M., Paul, J. L., Rothenberg, M. E., Marcellin, P., Durand, F., Bedossa, P., Prip-Buus, C., Bauge, E., Staels, B., Boulanger, C. M., Tedgui, A., \& Rautou, P. E. (2016). Liver microRNA-21 is overexpressed in non-alcoholic steatohepatitis and contributes to the disease in experimental models by inhibiting PPARalpha expression. Gut, 65(11), 1882-1894. doi:10.1136/gutjnl-2014-308883

Makki, K., Froguel, P., \& Wolowczuk, I. (2013). Adipose tissue in obesity-related inflammation and insulin resistance: cells, cytokines, and chemokines. ISRN Inflamm, 2013, 139239. doi:10.1155/2013/139239

Martin, G., Schoonjans, K., Lefebvre, A. M., Staels, B., \& Auwerx, J. (1997). Coordinate regulation of the expression of the fatty acid transport protein and acyl-CoA synthetase genes by PPARalpha and PPARgamma activators. J Biol Chem, 272(45), 28210-28217.

Matthews, V. B., Allen, T. L., Risis, S., Chan, M. H., Henstridge, D. C., Watson, N., Zaffino, L. A., Babb, J. R., Boon, J., Meikle, P. J., Jowett, J. B., Watt, M. J., Jansson, J. O., Bruce, C. R., \& Febbraio, M. A. (2010). Interleukin-6-deficient mice develop hepatic inflammation and systemic insulin resistance. Diabetologia, 53(11), 2431-2441.

Mauer, J., Chaurasia, B., Goldau, J., Vogt, M. C., Ruud, J., Nguyen, K. D., Theurich, S., Hausen, A. C., Schmitz, J., Bronneke, H. S., Estevez, E., Allen, T. L., Mesaros, A., Partridge, L., Febbraio, M. A., Chawla, A., Wunderlich, F. T., \& Bruning, J. C. (2014). Signaling by IL6 promotes alternative activation of macrophages to limit endotoxemia and obesityassociated resistance to insulin. Nat Immunol, 15(5), 423-430. doi:10.1038/ni.2865

Mishra, D., Richard, J. E., Maric, I., Porteiro, B., Haring, M., Kooijman, S., Musovic, S., Eerola, K., Lopez-Ferreras, L., Peris, E., Grycel, K., Shevchouk, O. T., Micallef, P., Olofsson, C. S., Wernstedt Asterholm, I., Grill, H. J., Nogueiras, R., \& Skibicka, K. P. (2019). Parabrachial Interleukin-6 Reduces Body Weight and Food Intake and Increases Thermogenesis to Regulate Energy Metabolism. Cell Rep, 26(11), 3011-3026 e3015. doi:10.1016/j.celrep.2019.02.044

Moh, A., Iwamoto, Y., Chai, G. X., Zhang, S. S., Kano, A., Yang, D. D., Zhang, W., Wang, J., Jacoby, J. J., Gao, B., Flavell, R. A., \& Fu, X. Y. (2007). Role of STAT3 in liver regeneration: survival, DNA synthesis, inflammatory reaction and liver mass recovery. Lab Invest, 87(10), 1018-1028. doi:10.1038/labinvest.3700630

Montagner, A., Polizzi, A., Fouche, E., Ducheix, S., Lippi, Y., Lasserre, F., Barquissau, V., Regnier, M., Lukowicz, C., Benhamed, F., Iroz, A., Bertrand-Michel, J., Al Saati, T., Cano, P., Mselli-Lakhal, L., Mithieux, G., Rajas, F., Lagarrigue, S., Pineau, T., Loiseau, N., Postic, C., Langin, D., Wahli, W., \& Guillou, H. (2016). Liver PPARalpha is crucial for wholebody fatty acid homeostasis and is protective against NAFLD. Gut, 65(7), 1202-1214. doi:10.1136/gutjnl-2015-310798

Narazaki, M., Yasukawa, K., Saito, T., Ohsugi, Y., Fukui, H., Koishihara, Y., Yancopoulos, G. D., Taga, T., \& Kishimoto, T. (1993). Soluble forms of the interleukin-6 signal-transducing receptor component gp130 in human serum possessing a potential to inhibit signals through membrane-anchored gp130. Blood, 82(4), 1120-1126.

Nikolajuk, A., Kowalska, I., Karczewska-Kupczewska, M., Adamska, A., Otziomek, E., Wolczynski, S., Kinalska, I., Gorska, M., \& Straczkowski, M. (2010). Serum soluble 
glycoprotein 130 concentration is inversely related to insulin sensitivity in women with polycystic ovary syndrome. Diabetes, 59(4), 1026-1029. doi:10.2337/db09-1316

Pasquin, S., Sharma, M., \& Gauchat, J. F. (2016). Cytokines of the LIF/CNTF family and metabolism. Cytokine, 82, 122-124. doi:10.1016/j.cyto.2015.12.019

Pedersen, B. K., \& Febbraio, M. A. (2008). Muscle as an endocrine organ: focus on muscle-derived interleukin-6. Physiol Rev, 88(4), 1379-1406. doi:10.1152/physrev.90100.2007

Rabe, B., Chalaris, A., May, U., Waetzig, G. H., Seegert, D., Williams, A. S., Jones, S. A., RoseJohn, S., \& Scheller, J. (2008). Transgenic blockade of interleukin 6 transsignaling abrogates inflammation. Blood, 111(3), 1021-1028. doi:blood-2007-07-102137 [pii] 10.1182/blood-2007-07-102137

Rose-John, S., Scheller, J., Elson, G., \& Jones, S. A. (2006). Interleukin-6 biology is coordinated by membrane-bound and soluble receptors: role in inflammation and cancer. J Leukoc Biol, 80(2), 227-236.

Schobitz, B., de Kloet, E. R., Sutanto, W., \& Holsboer, F. (1993). Cellular localization of interleukin 6 mRNA and interleukin 6 receptor mRNA in rat brain. Eur J Neurosci, 5(11), 1426-1435.

Timper, K., Denson, J. L., Steculorum, S. M., Heilinger, C., Engstrom-Ruud, L., Wunderlich, C. M., Rose-John, S., Wunderlich, F. T., \& Bruning, J. C. (2017). IL-6 Improves Energy and Glucose Homeostasis in Obesity via Enhanced Central IL-6 trans-Signaling. Cell Rep, 19(2), 267-280. doi:10.1016/j.celrep.2017.03.043

van Diepen, J. A., Jansen, P. A., Ballak, D. B., Hijmans, A., Hooiveld, G. J., Rommelaere, S., Galland, F., Naquet, P., Rutjes, F. P., Mensink, R. P., Schrauwen, P., Tack, C. J., Netea, M. G., Kersten, S., Schalkwijk, J., \& Stienstra, R. (2014). PPAR-alpha dependent regulation of vanin-1 mediates hepatic lipid metabolism. J Hepatol, 61(2), 366-372. doi:10.1016/j.jhep.2014.04.013

Vida, M., Serrano, A., Romero-Cuevas, M., Pavon, F. J., Gonzalez-Rodriguez, A., Gavito, A. L., Cuesta, A. L., Valverde, A. M., Rodriguez de Fonseca, F., \& Baixeras, E. (2013). IL-6 cooperates with peroxisome proliferator-activated receptor-alpha-ligands to induce liver fatty acid binding protein (LFABP) up-regulation. Liver Int, 33(7), 1019-1028. doi:10.1111/liv.12156

Wallenius, V., Wallenius, K., Ahren, B., Rudling, M., Carlsten, H., Dickson, S. L., Ohlsson, C., \& Jansson, J. O. (2002). Interleukin-6-deficient mice develop mature-onset obesity. Nat Med, 8(1), 75-79. doi:10.1038/nm0102-75

Wedell-Neergaard, A. S., Lang Lehrskov, L., Christensen, R. H., Legaard, G. E., Dorph, E., Larsen, M. K., Launbo, N., Fagerlind, S. R., Seide, S. K., Nymand, S., Ball, M., Vinum, N., Dahl, C. N., Henneberg, M., Ried-Larsen, M., Nybing, J. D., Christensen, R., Rosenmeier, J. B., Karstoft, K., Pedersen, B. K., Ellingsgaard, H., \& Krogh-Madsen, R. (2018). ExerciseInduced Changes in Visceral Adipose Tissue Mass Are Regulated by IL-6 Signaling: A Randomized Controlled Trial. Cell Metab, 844-855. doi:10.1016/j.cmet.2018.12.007

Weiss, T. W., Arnesen, H., \& Seljeflot, I. (2013). Components of the interleukin-6 transsignalling system are associated with the metabolic syndrome, endothelial dysfunction and arterial stiffness. Metabolism, 62(7), 1008-1013. doi:10.1016/j.metabol.2013.01.019

Wexler, H. M. (2007). Bacteroides: the good, the bad, and the nitty-gritty. Clin Microbiol Rev, 20(4), 593-621. doi:10.1128/CMR.00008-07

Wonnerth, A., Katsaros, K. M., Krychtiuk, K. A., Speidl, W. S., Kaun, C., Thaler, K., Huber, K., Wojta, J., Maurer, G., Seljeflot, I., Arnesen, H., \& Weiss, T. W. (2014). Glycoprotein 130 polymorphism predicts soluble glycoprotein 130 levels. Metabolism, 63(5), 647-653. doi:10.1016/j.metabol.2014.02.005

Woting, A., Pfeiffer, N., Loh, G., Klaus, S., \& Blaut, M. (2014). Clostridium ramosum promotes high-fat diet-induced obesity in gnotobiotic mouse models. mBio, 5(5), e01530-01514. doi:10.1128/mBio.01530-14

Wunderlich, F. T., Strohle, P., Konner, A. C., Gruber, S., Tovar, S., Bronneke, H. S., JunttiBerggren, L., Li, L. S., van Rooijen, N., Libert, C., Berggren, P. O., \& Bruning, J. C. (2010). Interleukin-6 signaling in liver-parenchymal cells suppresses hepatic inflammation and improves systemic insulin action. Cell Metab, 12(3), 237-249. 
Zhang, Q., Wang, H. Y., Liu, X., Bhutani, G., Kantekure, K., \& Wasik, M. (2011). IL-2R common gamma-chain is epigenetically silenced by nucleophosphin-anaplastic lymphoma kinase (NPM-ALK) and acts as a tumor suppressor by targeting NPM-ALK. Proc Natl Acad Sci U S A, 108(29), 11977-11982. doi:10.1073/pnas.1100319108

Zuliani, G., Galvani, M., Maggio, M., Volpato, S., Bandinelli, S., Corsi, A. M., Lauretani, F., Cherubini, A., Guralnik, J. M., Fellin, R., \& Ferrucci, L. (2010). Plasma soluble gp130 levels are increased in older subjects with metabolic syndrome. The role of insulin resistance. Atherosclerosis, 213(1), 319-324. doi:10.1016/j.atherosclerosis.2010.08.074 


\section{Figure Legends}

Figure 1. Sgp130-mediated trans-signaling blockade induces mature-onset obesity in mice on a normal chow diet.

(A) Photographs showing representative male, sgp130Fc and WT mice at 14 months of age.

(B) Body weight progression from 2 to 14 months of age in male, sgp130Fc and WT mice (n=920). The primary observation repeated in 4 independent experiments in male littermates and once in both male and female non-littermate mice.

(C) Representative coronal MRI images of sgp130Fc and WT mice at 10 months.

(D) Quantification of subcutaneous and abdominal fat tissue in mice from MRI images ( $n=4-5$ )

(E) Photographic image of representative gonadal fat pads and quantification of fat pad mass (right) at 14 months $(n=12)$.

(F) EchoMRI quantification of fat mass at 14 months (n=9-10).

Data are mean $\pm \mathrm{SD}(\mathrm{E}-\mathrm{F})$, or mean \pm SEM $(\mathrm{B}) . * * \mathrm{P}<0.01$, and $* * * \mathrm{P}<0.001$, by two tailed, Student's t test. (See also Figure S1.)

Figure 2. Peripheral trans-signaling blockade induces metabolic and behavioral changes in mice.

(A) Serum leptin concentrations at 2, 7, and 12 months (mths) (n=5-6).

(B) Daily food-intake at 3, 4 and 14 months of age $(n=6-7)$. Data represent mean food intake per mouse/cage per day averaged over a 2-week period.

(C) Western blot analysis and quantification (right) of Stat3 phosphorylation in the hypothalamus of 3.5 month old mice treated with leptin or carrier control $(n=2-4)$.

(D) Metabolic cage assessment of dark cycle total energy expenditure (TEE) and voluntary physical activity (Wheel Running) of mice at 4 and 13 months of age $(n=12)$.

Data are represented as mean \pm SD. $* P<0.05$, $* * P<0.01$, $* * * P<0.001$, and ${ }^{* * * * *} P<0.0001$ by two-tailed, Student's t test $(\mathrm{G}, \mathrm{H})$, or by two-way ANOVA (C,D). Significant interactions were reported for leptin induced p-STAT3 and genotype $(P=0.0193)$, and for genotype and age for TEE $(* * \mathrm{P}=0.0024)$ and Wheel Running $(* * * * P<0.0001)$. (See also Figure S1.) 
Figure 3. IL-6 trans-signaling blockade induces adipocyte hypertrophy and ATM recruitment.

(A) $\mathrm{H} \& \mathrm{E}$ stained adipose tissue sections in $\mathrm{sgp} 130 \mathrm{Fc}$ mice and WT littermates at 14 months of age. Scale bars, $20 \mu \mathrm{m}$.

(B) Quantification of adipocytes with cell cross-sectional area $>6000$ square pixels by ImageJ, $(n=4-7)$.

(C) Photomicrograph of F4/80 immunostaining of adipose tissue thin sections showing adipose tissue-associated macrophage (ATM) (red) in crown-like structures (CLS) (arrows) at 14 months. Scale bars, $20 \mu \mathrm{m}$.

(D) Quantification of F4/80+ CLS per high power field (HPF) in adipose tissue thin sections (c), $(n=4)$.

(E) Representative flow cytometry plots (left) and quantification (right) of $\mathrm{F} 4 / 80^{+}$cells in adipose tissue of 11-month old mice, $(n=3-4)$. F4/80+ cells were quantified as per the bottom right quadrant (gate P2) (red).

Data are represented as mean $\pm \mathrm{SD} .{ }^{*} \mathrm{P}<0.05$, and $* * \mathrm{P}<0.01$ by two-tailed, Mann Whitney test (B), or two-tailed, Student's t test (D, E). (See also Figure S2.)

Figure 4. Inhibition of IL-6 trans-signaling induces glucose intolerance and peripheral insulin resistance.

(A) Fasting glucose levels in sgp130Fc and WT littermates ( $n=7)$.

(B) Glucose levels before and after glucose challenge in sgp130Fc versus WT mice at 5 (left) and 12 (right) months of age $(n=7)$.

(C) Blood glucose levels in fasting 12-month-old sgp130Fc and WT mice at indicated times following insulin injection (i.p.) (n=7).

(D) Fasting and postprandial serum insulin levels in sgp $130 \mathrm{Fc}$ and WT littermates at 13 months of age $(n=5)$.

Data are represented as mean $\pm \mathrm{SD}(\mathrm{A}, \mathrm{D})$, or mean $\pm \mathrm{SEM}(\mathrm{B}, \mathrm{C}) . * P<0.05, * * P<0.01$, and $* * *$ $P<0.001$ by two-tailed Student's $t$ test. (B,C) $* P<0.05$, ** $P<0.01$ for comparisons of area under the curve by two-tailed Student's $t$ test. (See also Figure S3.)

Figure 5. Inhibition of trans-signaling induces steatosis without hepatic inflammation.

(A) Representative images showing fat accumulation by oil red O (ORO) staining in livers in sgp130Fc and WT littermates at the age of 14 months and ImageJ processed image (Inset). Scale bars represent $20 \mu \mathrm{m}$. (right) Quantification of ORO-stained area by Image J, (n=4). 
(B) Representative plots from flow cytometry of double-positive Cd45+/Cd11b+ and $\mathrm{Cd} 45+/ \mathrm{F} 4 / 80+$ cells (upper right quadrant) in livers of sgp130Fc and WT littermates at 11 months of age and quantification (right), (n=8-9).

Data are represented as mean $\pm \mathrm{SD} . * \mathrm{P}<0.05, * * * \mathrm{P}<0.001$ by two-tailed, Student's $t$ test. (See also Figures S2 and S5.)

Figure 6. Suppression of hepatic PPAR $\alpha$ and miR-21 upregulation are associated with IL-6 trans-signaling blockade.

(A) Phosphorylated Stat3 and $\beta$-Actin protein levels by Western Blot analysis of liver samples from 5-month old sgp $130 \mathrm{Fc}$ and WT littermates and quantification of band intensities (right). $(n=5-7)$.

(B) Hepatic miR-21 and miR-122 levels in sgp130Fc and WT littermates at 2 and 14 months of age by real time qPCR analysis, $(n=5-6)$.

(C) Real time qPCR analysis of hepatic mRNAs encoding Ppara, and PPAR $\alpha$ targets: Cpt-1, Apoe, and Fasn, in sgp130Fc and WT littermates at 2 and 14 months of age $(\mathrm{n}=5)$.

(D) Western blot analysis of PPAR $\alpha$ and $\beta$-Actin protein levels in liver samples from 5 months old sgp130Fc mice and WT littermates with quantification of band intensity by Image J (right), $(n=4-7)$.

Data are represented as mean $\pm \mathrm{SD}$. $* P<0.05$, ** $P<0.01$, and $* * * P<0.001$ by two-tailed, Student's $t$ test. (See also Figure S6.)

Figure 7. PPARa agonist fenofibrate reverses the mature-onset metabolic phenotype in sgp130Fc mice.

(A) Schematic representation of the experimental design. Sgp130Fc and WT littermates aged 4.5 months were administered a Normal Chow Diet (NCD), or a NCD supplemented with Fenofibrate (FF) for 2.5 months. Fasting blood glucose levels and insulin tolerance (ITT) were assessed at 6.5 months, and the mice sacrificed at 7 months of age.

(B) Body weight (left) and gonadal fat pad (right) weight in 7 months old sgp130Fc and WT littermates following NCD and FF diets ( $\mathrm{n}=8-9)$.

(C) Photographs showing representative gonadal fat pads at 7 months of age from mice fed NCD or FF diets. Scale bars, $1 \mathrm{~cm}$.

(D) Fasting blood glucose levels in NCD or FF fed mice at the age of 6.5 months (n=6-8).

(E) Insulin tolerance test (ITT) at the age of 6.5 months in sgp130Fc and WT littermates treated with NCD or FF diets $(n=6-8)$.

Page $\mid 20$ 
(F) Microphotographs showing representative ORO-stained liver sections from 7 months old NCD or FF fed mice with ImageJ processed image (inset), and quantification (right) of oil red $\mathrm{O}$ (ORO)-stained area in Image J processed images $(n=4)$. Scale bars, $20 \mu \mathrm{m}$.

Numerical data are represented as mean $\pm \mathrm{SD}(\mathrm{B}, \mathrm{D}, \mathrm{F})$, or mean $\pm \mathrm{SEM}(\mathrm{E}) . * \mathrm{P}<0.05, * * \mathrm{P}<0.01$, and $* * * \mathrm{P}<0.001$ by two-way ANOVA (B,D,F); (E) *** $\mathrm{P}<0.001$ by two-way ANOVA for comparisons of area under the curve. Significant interaction between genotype and diet were reported for: B: Body weight $(* P=0.02)$, Gonadal Fat Pad weight $(* P=0.05)$, and $\mathrm{D}(* * P=0.008)$. (See also Figure S7.) 


\section{Figure 1}

A WT sgp130Fc

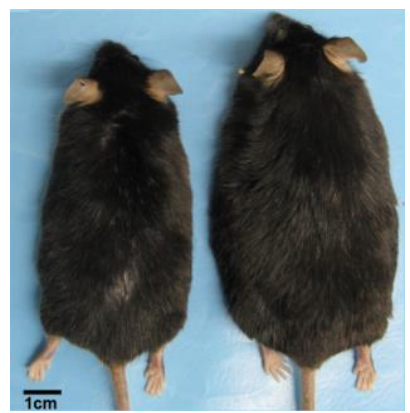

C

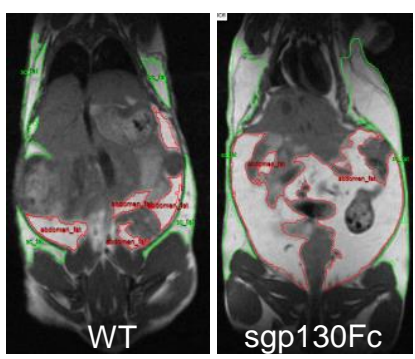

B

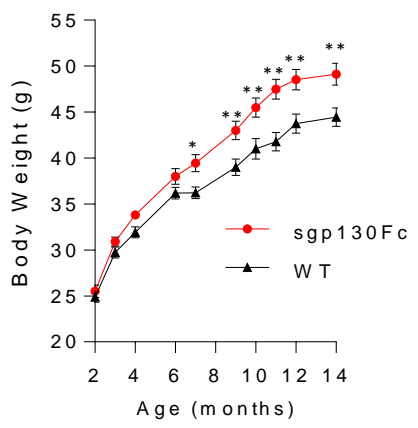

D

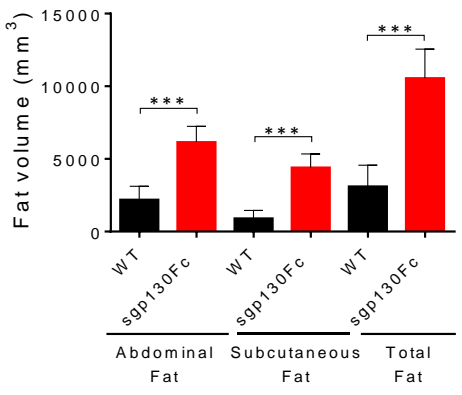

E
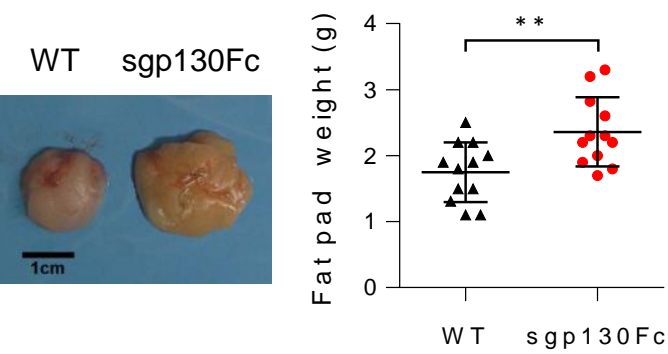

$\mathbf{F}$

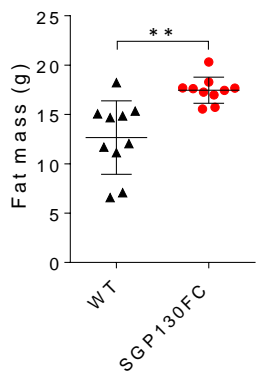

Figure 1. Sgp130-mediated trans-signaling blockade induces mature-onset obesity in mice on a normal chow diet.

(A) Photograph showing representative male, sgp130Fc and WT mice at 14 months of age.

(B) Body weight progression from 2 to 14 months of age in male, sgp130Fc and WT mice $(n=9-20)$. The primary observation repeated in 4 independent experiments in male littermates and once in both male and female non-littermate mice.

(C) Representative coronal MRI images of sgp130Fc and WT mice at 10 months.

(D) Quantification of subcutaneous and abdominal fat tissue in mice from MRI images $(n=4-5)$

(E) Photographic image of representative gonadal fat pads and quantification of fat pad mass (right) at 14 months ( $n=12)$.

(F) EchoMRI quantification of fat mass at 14 months $(n=9-10)$.

Data are represented as mean $\pm \mathrm{SD}(\mathrm{E}-\mathrm{F})$, or mean $\pm \mathrm{SEM}(\mathrm{B}) .{ }^{* *} p<0.01$, and ${ }^{* * *} p<0.001$, by two tailed, Student's $t$ test. (See also Figure S1.) 


\section{Figure 2}

A

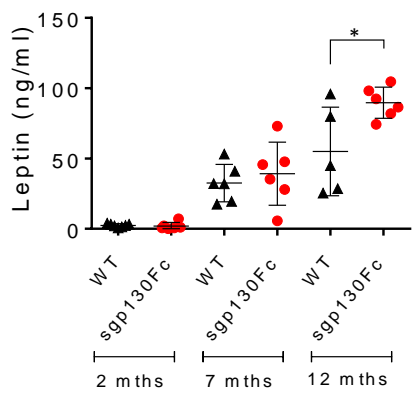

B

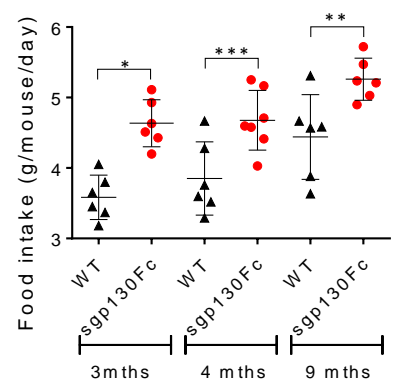

C
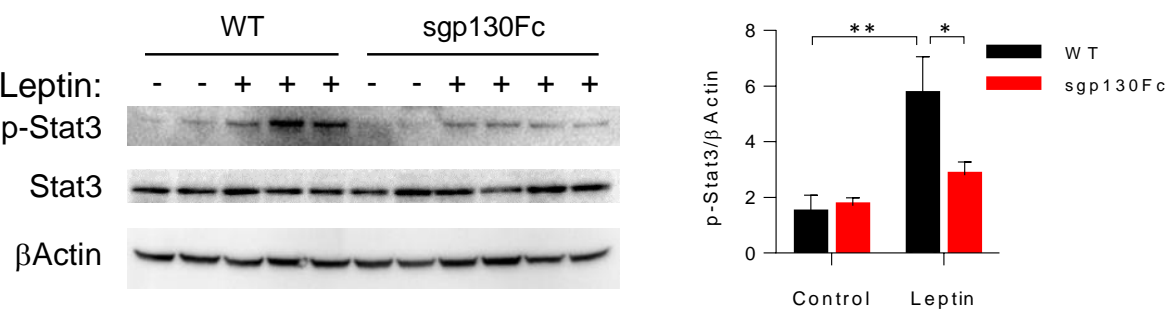

D
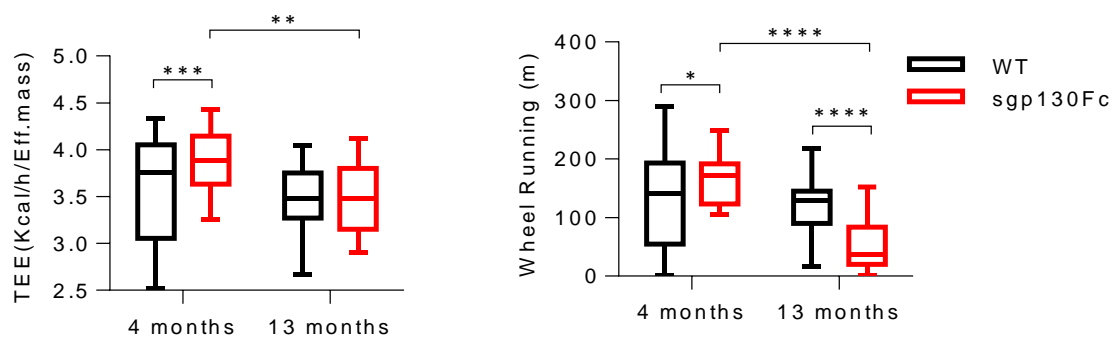

Figure 2. Peripheral trans-signaling blockade induces metabolic and behavioral changes in mice.

(A) Serum leptin concentrations at 2, 7, and 12 months (mths) ( $n=5-6)$.

(B) Daily food-intake at 3, 4 and 14 months of age $(n=6-7)$. Data represent mean food intake per mouse/cage per day averaged over a 2-week period.

(C) Western blot analysis and quantification (right) of Stat3 phosphorylation in the hypothalamus of 3.5 month old mice treated with leptin or carrier control $(n=2-4)$.

(D) Metabolic cage assessment of dark cycle total energy expenditure (TEE) and voluntary physical activity (Wheel Running) of mice at 4 and 13 months of age $(n=12)$.

Data are represented as mean \pm SD. ${ }^{*} P<0.05,{ }^{* *} P<0.01,{ }^{* * *} p<0.001$, and ${ }^{* * * *} p<0.0001$ by two-tailed, Student's t test $(G, H)$, or by two-way ANOVA $(C, D)$. Significant interactions were reported for leptin induced $p$-STAT3 and genotype $(P=0.0193)$, and for genotype and age for TEE $(* * P=0.0024)$ and Wheel Running $(* * * * P<0.0001)$. (See also Figure S1.) 


\section{Figure 3}

A WT

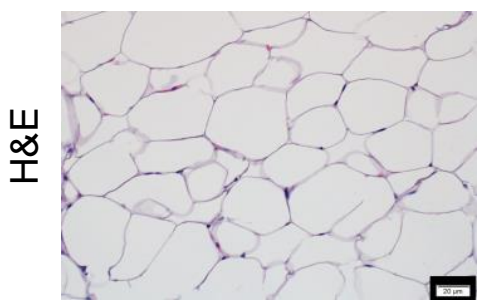

C

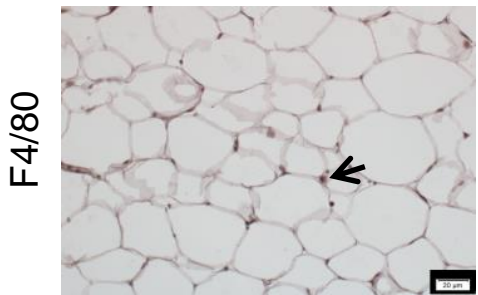

E

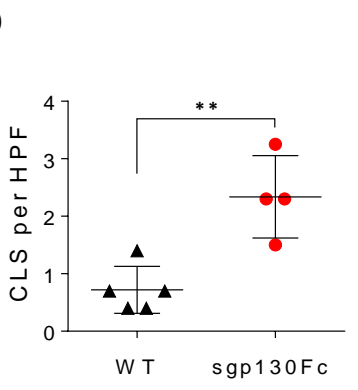

D
sgp130Fc
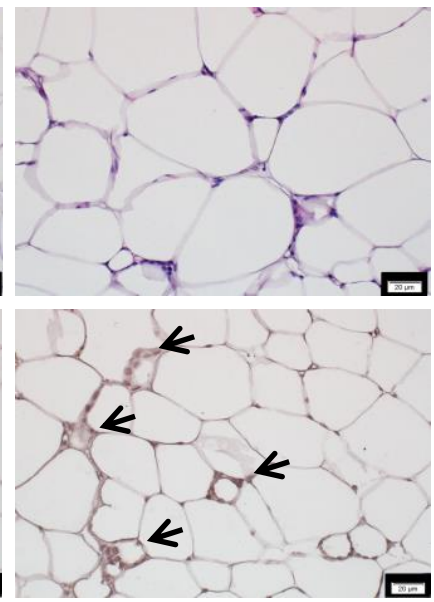

B

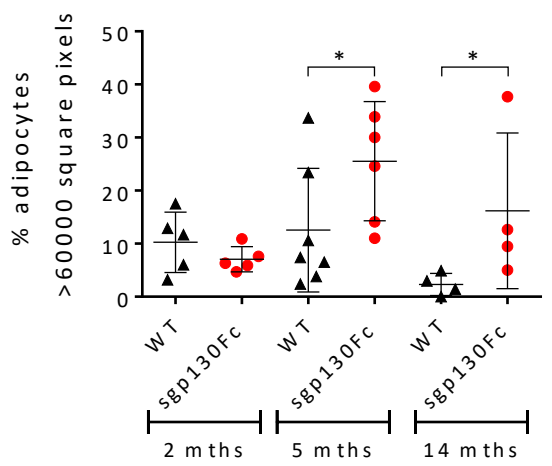

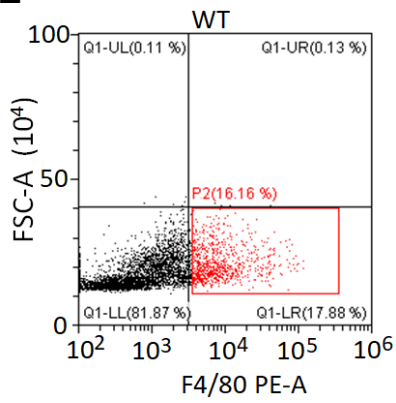
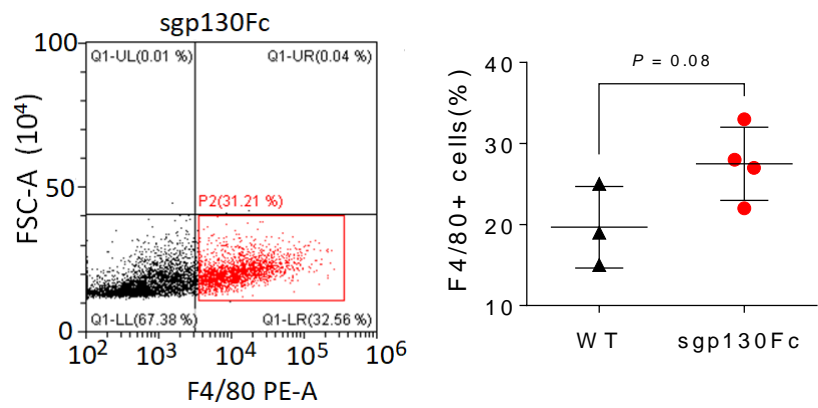

Figure 3. IL-6 trans-signaling blockade induces adipocyte hypertrophy and ATM recruitment.

(A) H\&E stained adipose tissue sections in sgp130Fc mice and WT littermates at 14 months of age. Scale bars, 20 $\mathrm{mm}$.

(B) Quantification of adipocytes with cell cross sectional area $>6000$ square pixels by ImageJ ( $n=4-7)$.

(C) Photomicrograph of F4/80 immunostaining of adipose tissue thin sections showing adipose tissue-associated macrophage (ATM) (red) in crown-like structures (CLS) (arrows) at 14 months. Scale bars, 20um.

(D) Quantification of F4/80+ CLS per high power field (HPF) in adipose tissue thin sections (c) $(n=4)$.

(E) Representative flow cytometry plots (left) and quantification (right) of F4/80-positive cells in adipose tissue of 11month old mice, ( $n=3-4)$. F4/80+ cells were quantified as per the bottom right quadrant (gate P2) (red).

Data are represented as mean \pm SD. ${ }^{*} \mathrm{P}<0.05$, and ${ }^{* *} \mathrm{P}<0.01$ by two-tailed, Mann Whitney test (B), or two-tailed, Student's $t$ test $(D, E)$. See also Figure S2. 


\section{Figure 4}

A

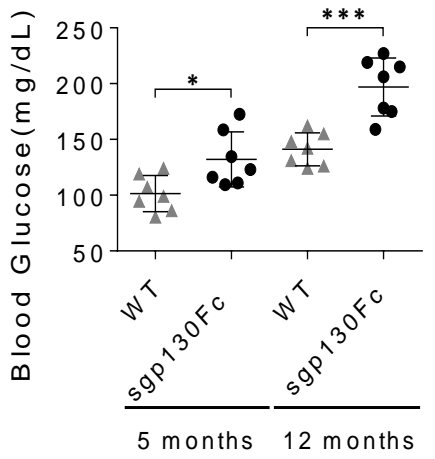

C

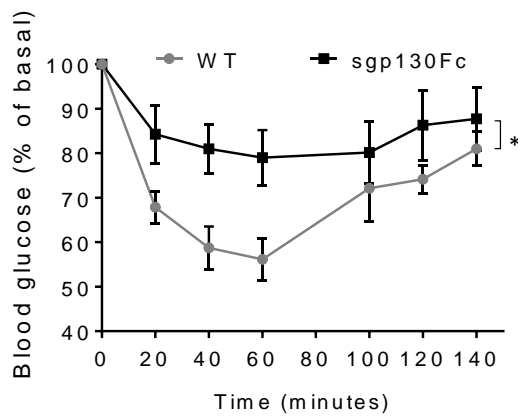

B

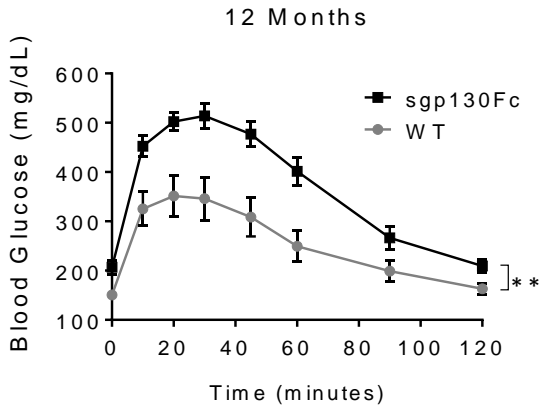

D

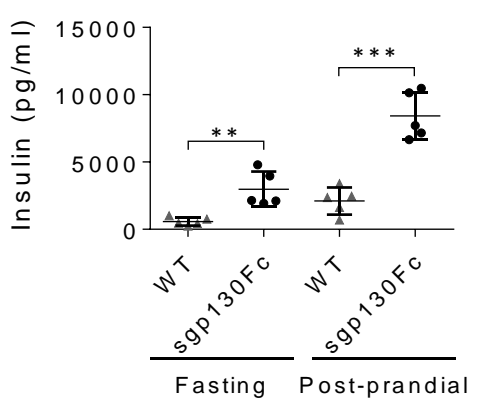

Figure 4. Inhibition of IL-6 trans-signaling induces glucose intolerance and peripheral insulin resistance.

(A) Fasting glucose levels in sgp130Fc and WT littermates $(n=7)$.

(B) Glucose levels before and after glucose challenge in sgp130Fc versus WT mice at 12 (right) months of age $(n=7)$.

(C) Blood glucose levels in fasting 12 month old sgp130Fc and WT mice at indicated times following insulin injection (i.p.) $(n=7)$.

(D) Fasting and post-prandial serum insulin levels in sgp130Fc and WT littermates at 13 months of age $(n=5)$.

Data are represented as mean \pm SD $(A, D)$, or mean \pm SEM $(B, C) .{ }^{*} P<0.05, * * P<0.01$, and ${ }^{* * *} P<0.001$ by two-tailed Student's $t$ test. $(B, C) * P<0.05, * * P<0.01$ for comparisons of area under the curve by two-tailed Student's $t$ test. (See also Figure S3.) 


\section{Figure 5}

A
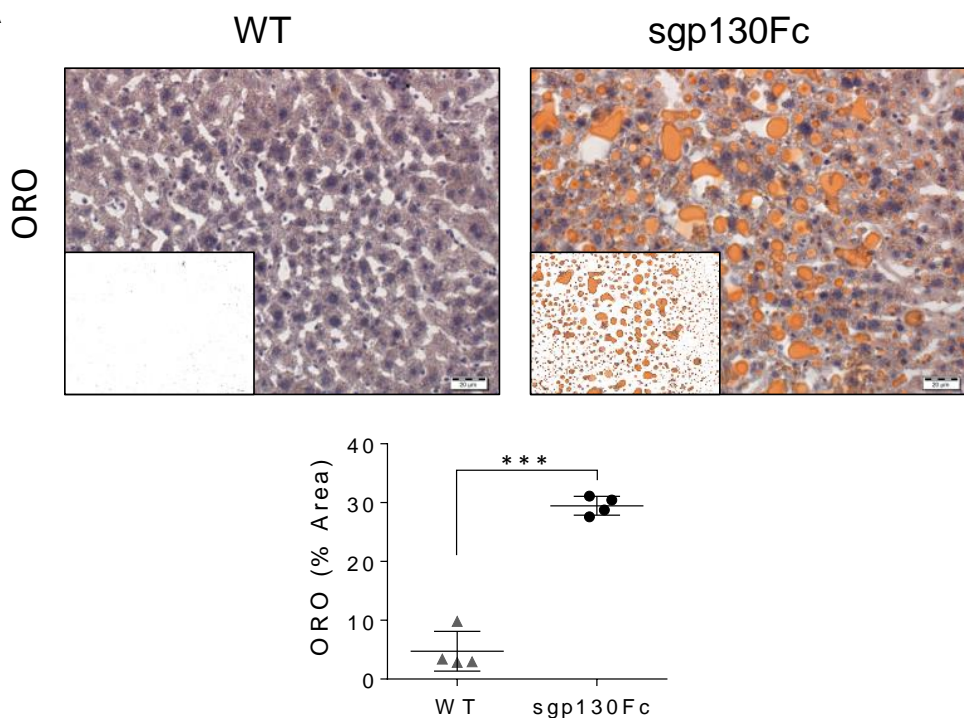

B
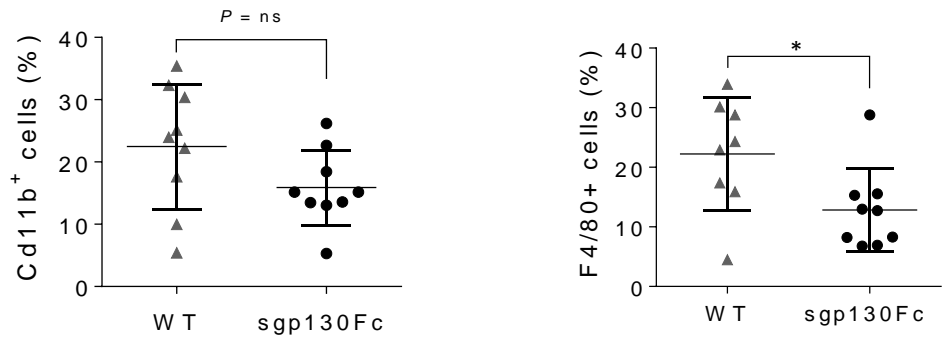

Figure 5. Inhibition of trans-signaling induces steatosis without hepatic inflammation.

(A) Representative images showing fat accumulation by oil red O (ORO) staining in livers in sgp130Fc and WT littermates at the age of 14 months and ImageJ processed image (Inset). Scale bars represent $20 \mu \mathrm{m}$. (below) Quantification of ORO-stained area by Image J, $(n=4)$.

(B) Representative plots from flow cytometry of double-positive Cd45+/Cd11b+ and Cd45+/F4/80+ cells (upper right quandrants) in livers of sgp130Fc and WT littermates at 11 months of age and quantification (right), $(n=9)$.

Data are represented as mean \pm SD. ${ }^{*} \mathrm{P}<0.05,{ }^{* * *} \mathrm{P}<0.001$ by two-tailed, Student's $t$ test. (See also Figures $\mathrm{S} 2$ and S5) 


\section{Figure 6}

A

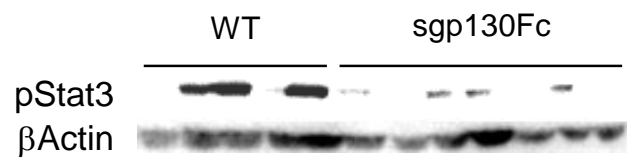

B

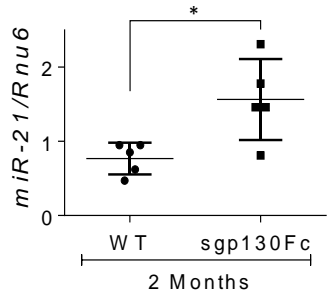

C
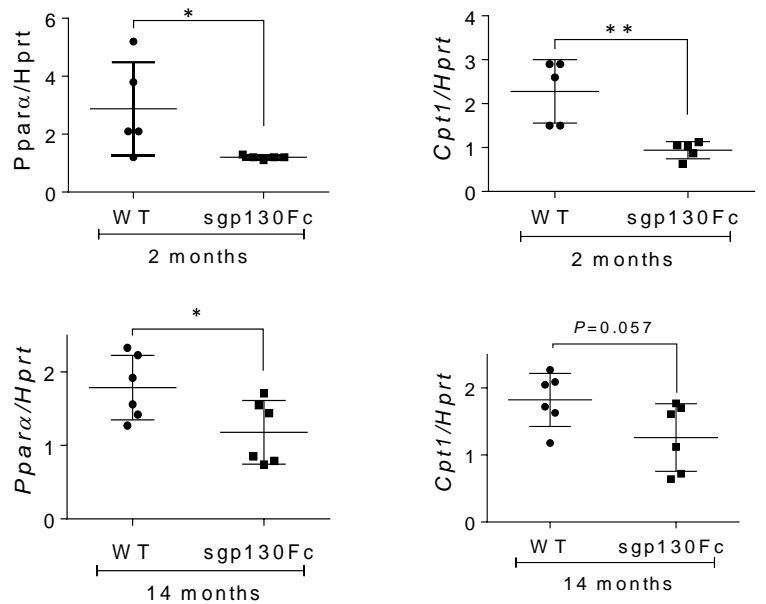

D

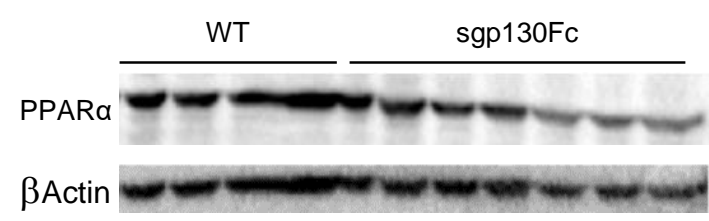

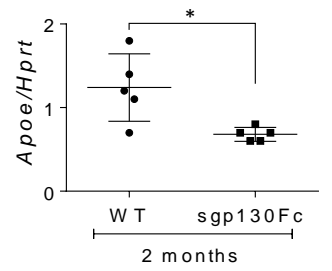
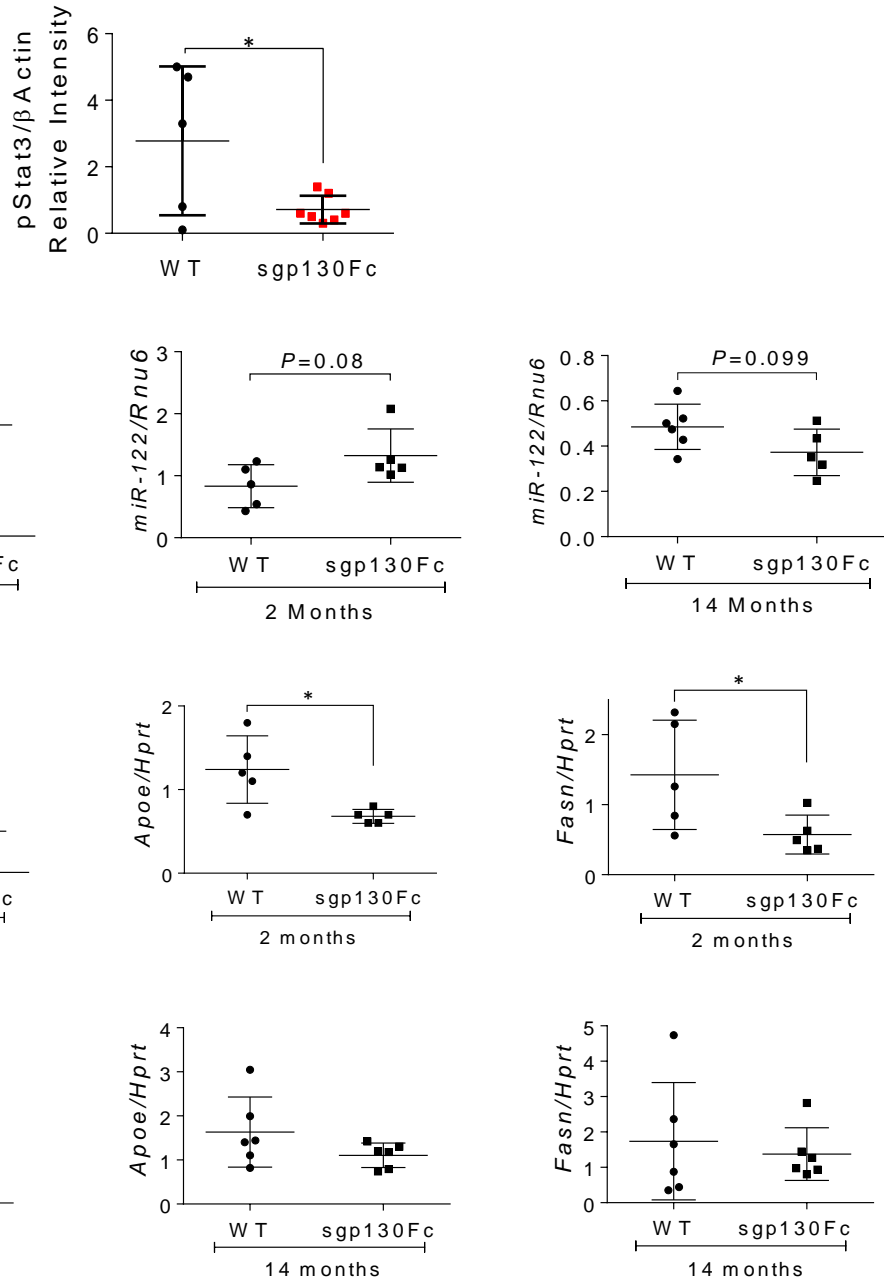

Figure 6. Suppression of hepatic PPAR $\alpha$ and miR-21 upregulation are associated with IL-6 trans-signaling blockade.

(A) Phosphorylated Stat3 and $\beta$-Actin protein levels by Western Blot analysis of liver samples from 5-month old sgp130Fc and WT littermates and quantification of band intensities (right), ( $n=5-7)$.

(B) Hepatic miR-21 and miR-122 levels in sgp130Fc and WT littermates at 2 and 14 months of age by real time qPCR analysis, $(n=5-6)$.

(C) Real time qPCR analysis of hepatic mRNAs encoding PPAR $\alpha$, and PPAR $\alpha$ targets: Cpt1, Apoe, and Fasn, in sgp130Fc and WT littermates at 2 and 14 months of age $(n=5)$.

(D) Western blot analysis of PPAR $\alpha$ and $\beta$-Actin protein levels in liver samples from 5 month old sgp130Fc mice and WT littermates with quantification of band intensity by Image J (right), ( $n=4-7)$.

Data are represented as mean \pm SD. ${ }^{*} \mathrm{P}<0.05,{ }^{* *} \mathrm{P}<0.01$, and ${ }^{* * *} \mathrm{P}<0.001$ by two-tailed, Student's $t$ test. 


\section{Figure 7}

A

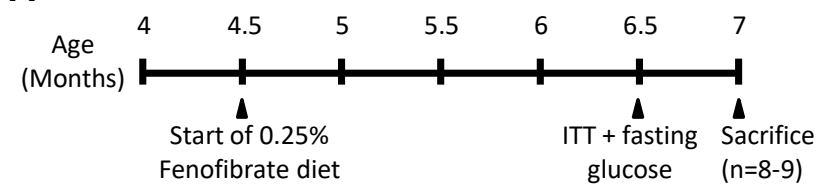

B

C
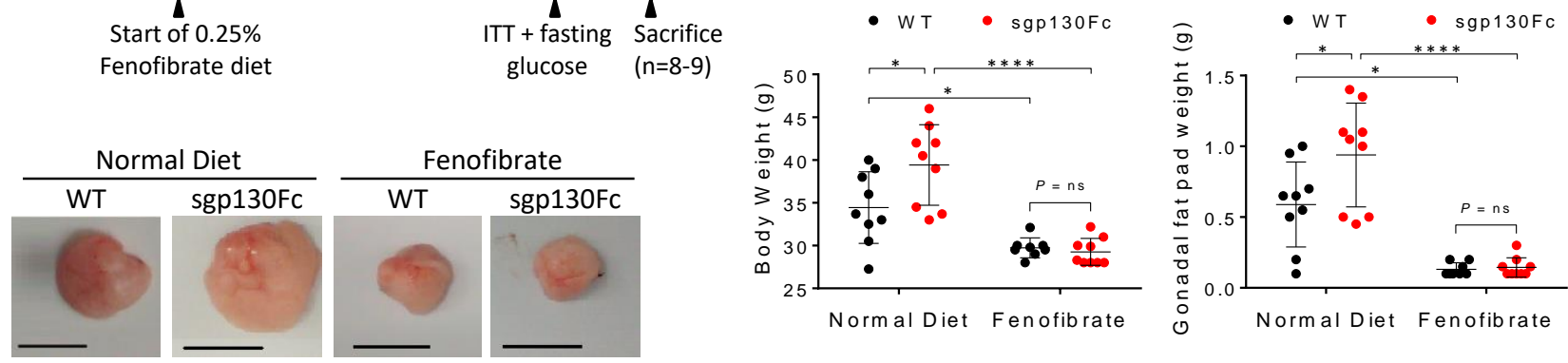

D

$\mathbf{E}$
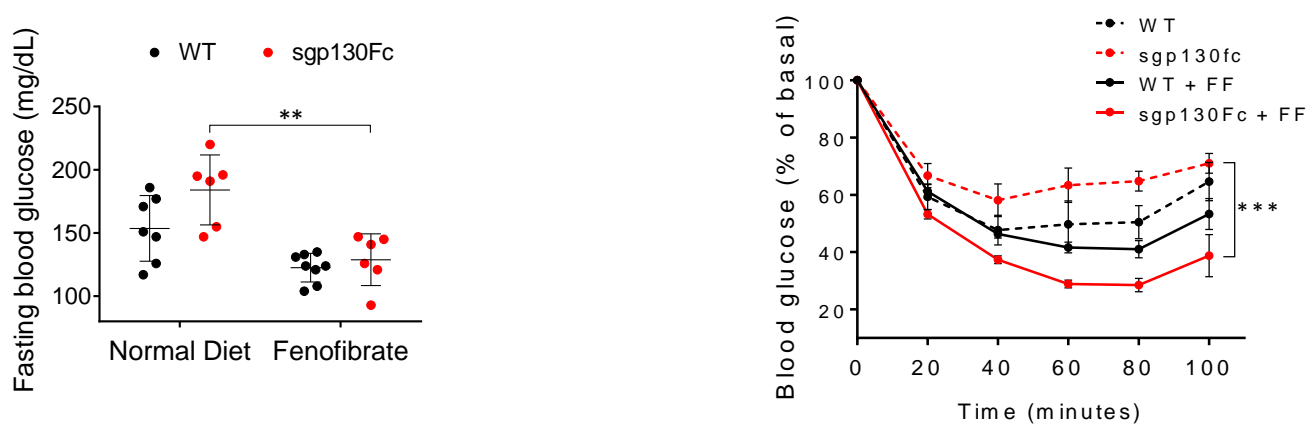

$\mathbf{F}$
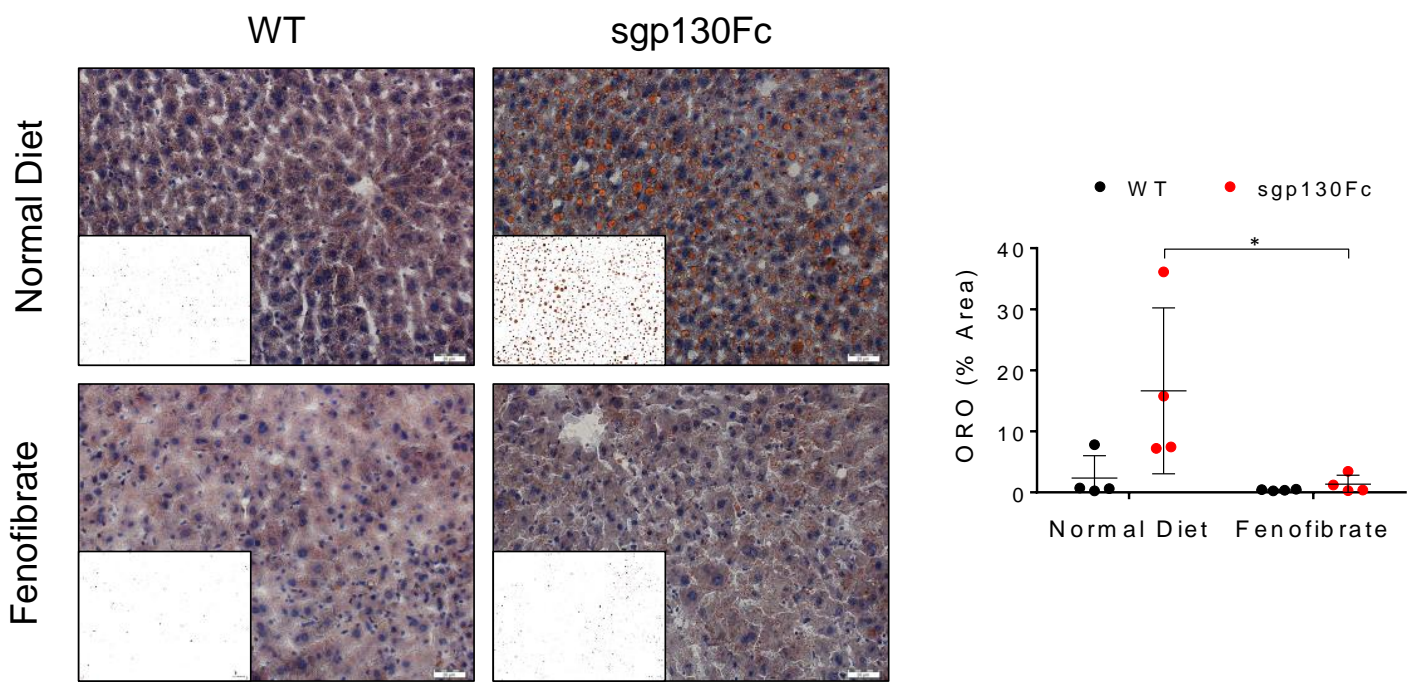

Figure 7. PPAR $\alpha$ agonist fenofibrate reverses the mature-onset metabolic phenotype in sgp130Fc mice.

(A) Schematic representation of the experimental design. Sgp130Fc and WT littermates aged 4.5 months were administered a Normal Chow Diet (NCD), or a NCD supplemented with Fenofibrate (FF) for 2.5 months. Fasting blood glucose levels and insulin tolerance (ITT) were assessed at 6.5 months, and the mice sacrificed at 7 months of age.

(B) Body weight (left) and Gonadal fat pad weight (right) in 7 month old sgp130Fc and WT littermates following NCD and FF diets $(n=8-9)$.

(C) Photographs showing representative gonadal fat pads at 7 months of age from mice fed NCD or FF diets. Scale bars, $1 \mathrm{~cm}$.

(D) Fasting blood glucose levels in NCD or FF fed mice at the age of 6.5 months $(n=6-8)$.

(E) Insulin tolerance test (ITT) at the age of 6.5 months in sgp130Fc and WT littermates treated with NCD or FF diets, $(n=6-8)$.

(F) Microphotographs showing representative ORO-stained liver sections from 7 month old NCD or FF fed mice with ImageJ processed image (inset), and quantification (right) of oil red O (ORO)-stained area in ImageJ processed images $(n=4)$. Scale bars, $20 \mu \mathrm{m}$.

Numerical data are represented as mean $\pm S D(B, C, E, G)$, or mean $\pm S E M(F) . * P<0.05, * * P<0.01$, and $* * * P<0.001$ by two-way ANOVA $(A, B, C, D, E, G) ;(F) * * * P<0.001$ by two-way ANOVA for comparisons of area under the curve. Significant interaction between genotype and diet were reported for: $\mathrm{B}\left({ }^{*} P=0.02\right), \mathrm{C}\left({ }^{*} P=0.05\right)$, and $\mathrm{F}\left({ }^{* *} P=0.008\right)$. 
Supplemental Figures 
Figure S1

A

C

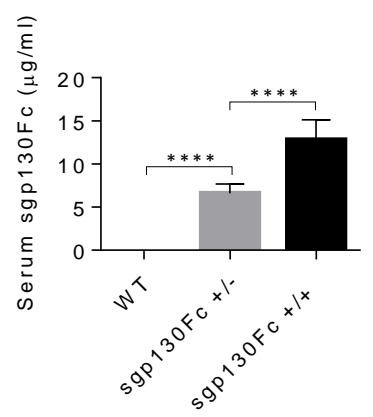

B

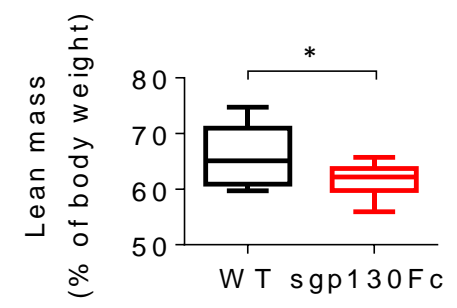

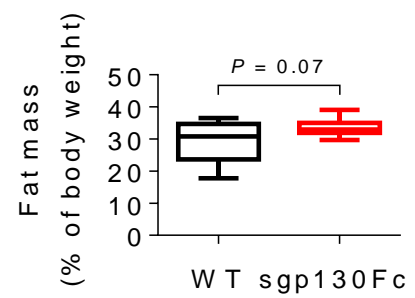
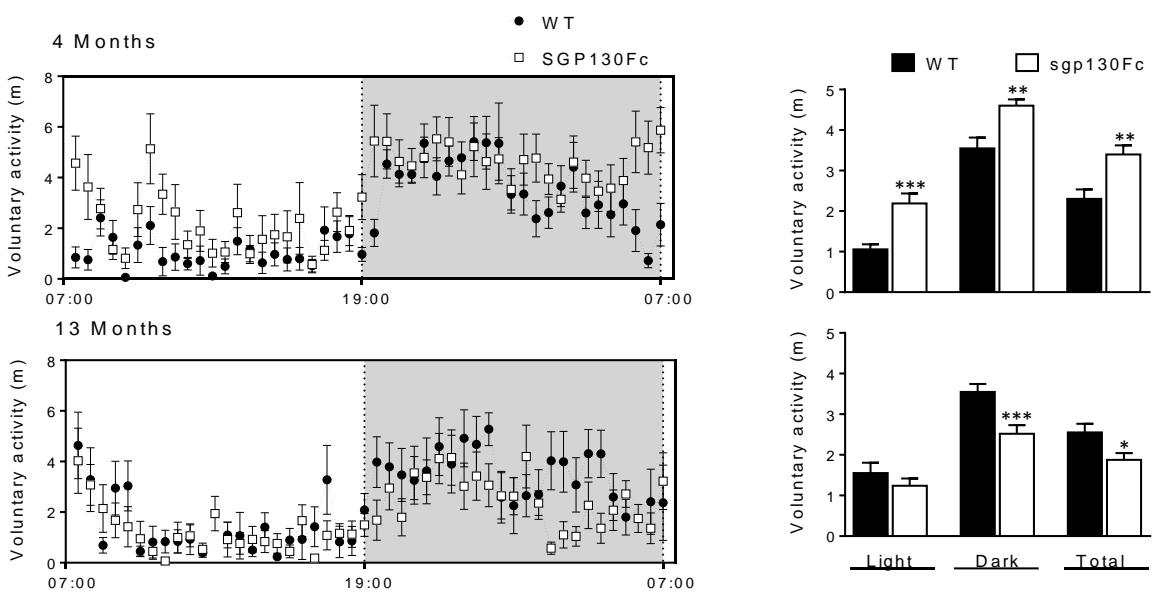

D
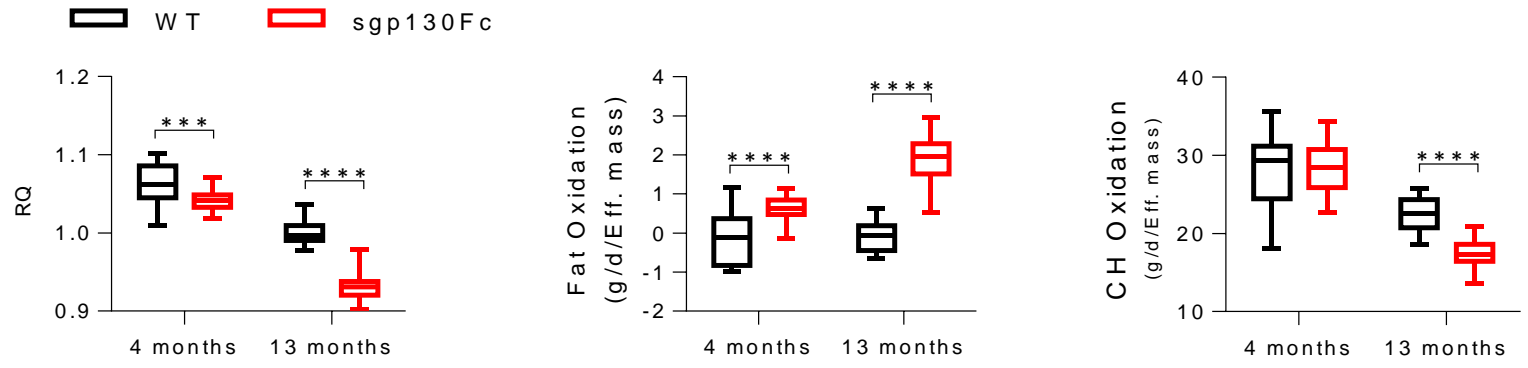

E
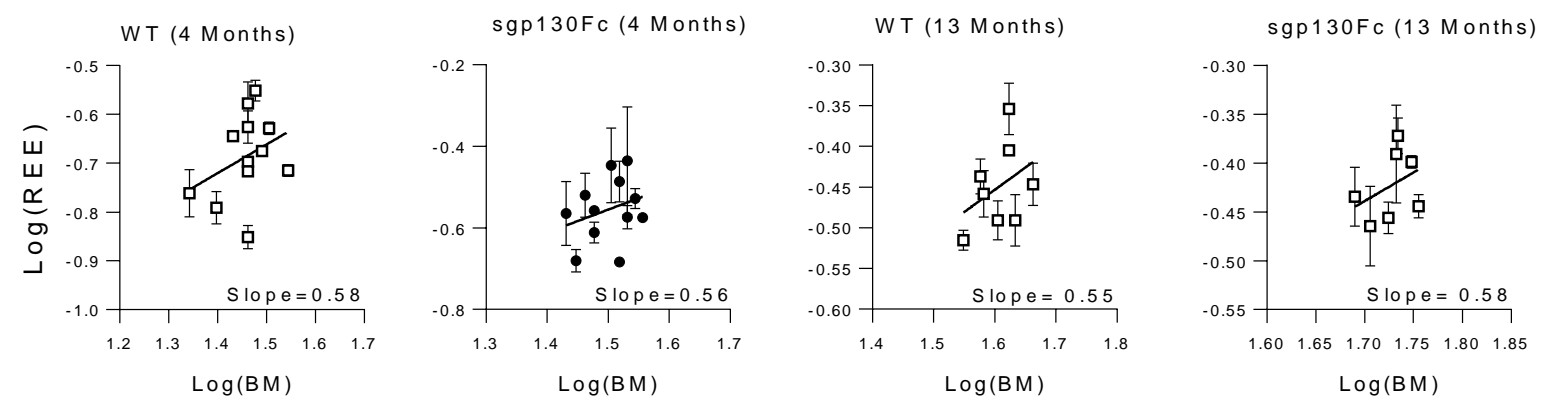
Figure S1 (Related to Figures 1 and 2) IL-6 trans-signaling blockade increases fat mass and decreases lean mass associated with decreased energy expenditure.

(A) Serum concentrations of human sgp130Fc by ELISA in wild type (WT), heterozygous $(-/+)$ and homozygous $(+/+)$ sgp130Fc mice at 2 months of age $(n=8-15)$.

(B) Echo MRI analysis of lean and fat body mass as a function of \% body weight in sgp130Fc and WT littermates at 14 months of age $(n=9-10)$.

(C) Voluntary activity (pedmeter) at $4(n=12)$ and $13(n=8)$ months of age.

(D) Metabolic activity for dark cycle including respiratory quotient $(R Q)$, fat oxidation and carbohydrate $(\mathrm{CH})$ oxidation of WT and sgp130Fc mice at $4(n=12)$ and 13 months $(n=8)$.

(E) Allometric regression analysis for determination of mass scaling exponent of WT and sgp130Fc mice.

Data are represented as mean $\pm \mathrm{SD}(\mathrm{A}, \mathrm{C})$, or box and whiskers plots $(\mathrm{B}, \mathrm{D}){ }^{*} P<0.05,{ }^{* \star} P<0.01,{ }^{\star *} P<0.01$ ${ }^{\star \star \star} P<0.001$, and ${ }^{* \star * \star} P<0.0001$ by two-tailed, Student's $t$ test (A-C), or by two-way ANOVA (D). Significant interactions $\left({ }^{\star \star \star \star} P<0.0001\right)$ between genotype and age were returned for $R Q$, fat oxidation and carbohydrate oxidation. 


\section{Figure S2}

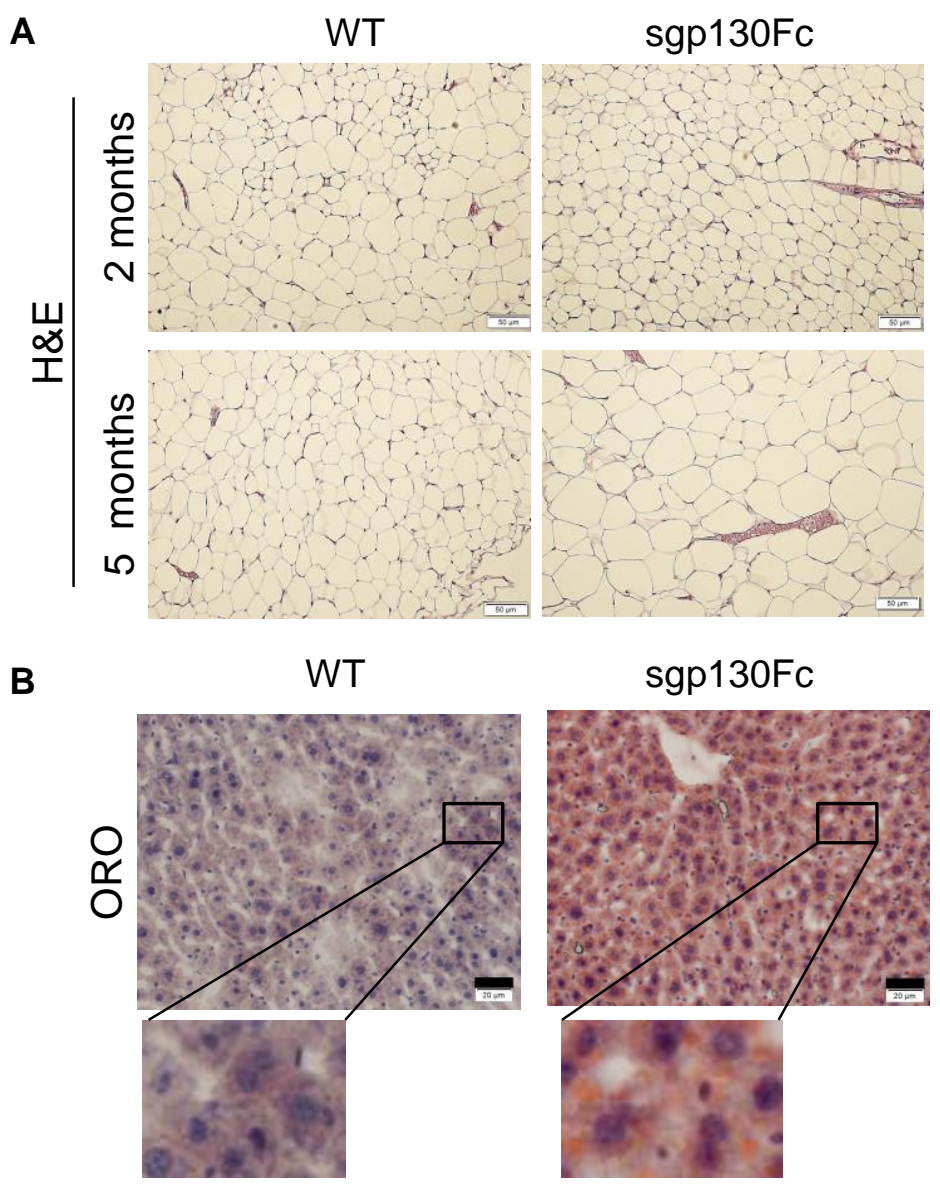

Figure S2 (Related to Figures 3 and 5). Inhibition of IL6 trans-signaling results in adipocyte hypertrophy and steatosis at 5 months of age

(A) H\&E stained sections from paraffin embedded adipose tissue from sgp130Fc and WT littermates at 2 and 5 months of age. Scale bars, $50 \mu \mathrm{m}$.

(B) ORO-stained liver sections from sgp130Fc and WT littermates at the age of 5months. Scale bars, $20 \mu \mathrm{m}$. 


\section{Figure S3}

A

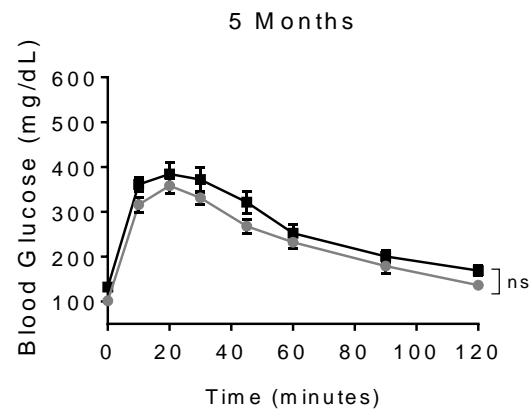

B
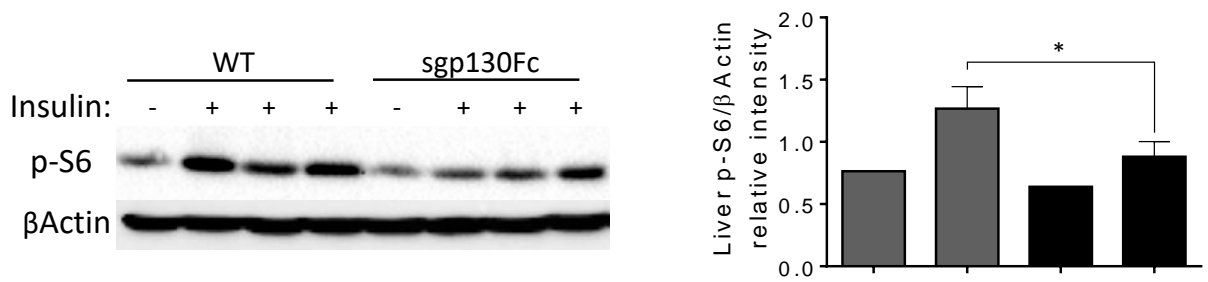

C
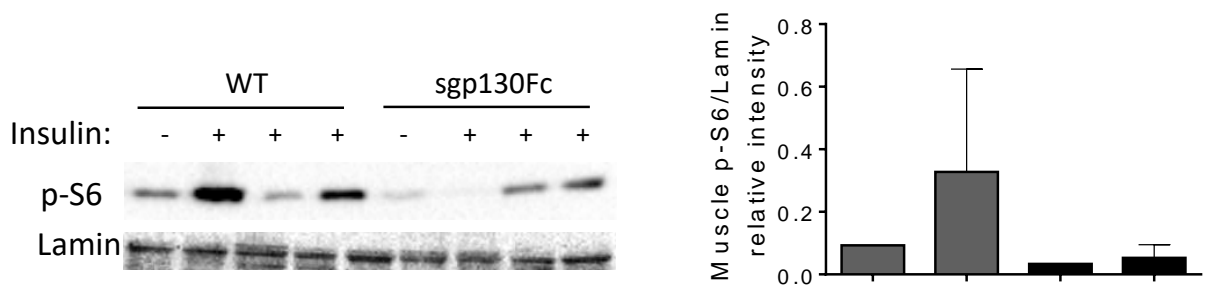

D

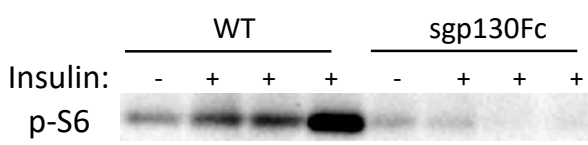

BActin

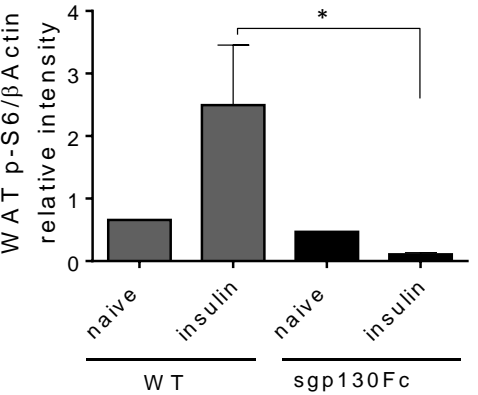

Figure S3 (Related to Figure 4). Inhibition of IL-6 trans-signaling induces glucose intolerance and peripheral insulin resistance.

(A) Glucose levels before and after glucose challenge in sgp130Fc versus WT mice at 5 (left) months of age $(n=7)$.

(B-D) Phospho-S6 (pS6) protein levels by Western Blot analysis of liver (B), muscle (C), and white adipose tissue (WAT) (D) tissues before and 10 minutes post insulin injection in 10 month-old sgp130Fc and WT mice.

Quantification of band intensity by ImageJ (right). ( $n=3$ for groups of insulin-treated mice).

Data are represented as mean \pm SEM (A), or mean \pm SD (B-D). (A) $P=n s$ for comparisons of area under the curve by two-tailed Student's $t$ test. $(B, D){ }^{*} P<0.05$, by two-tailed Student's $t$ test. 


\section{Figure S4}

A

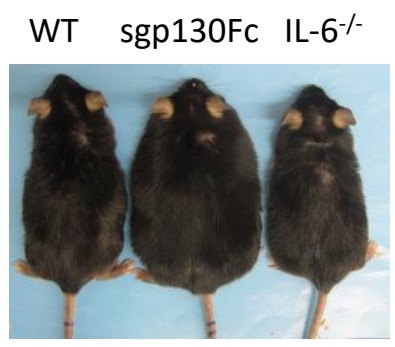

$\mathbf{E}$

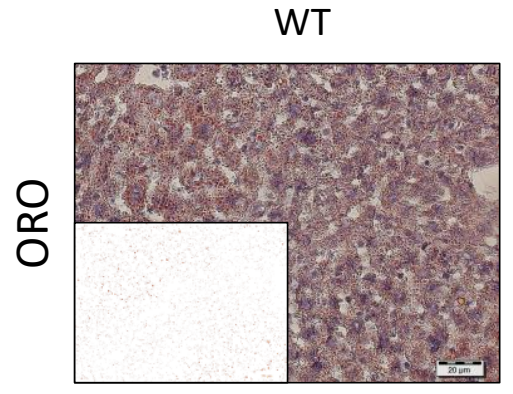

B

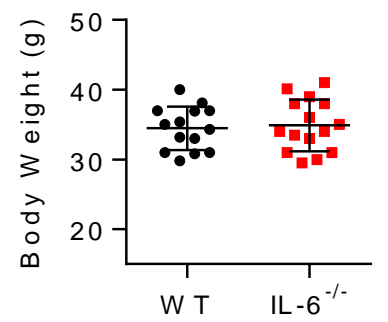

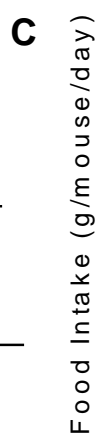

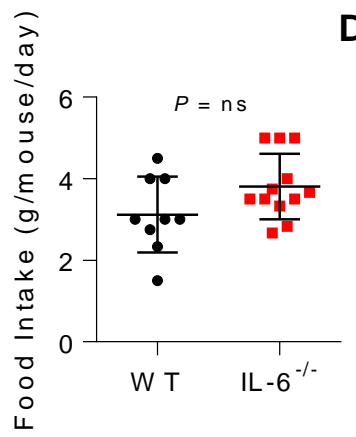

D

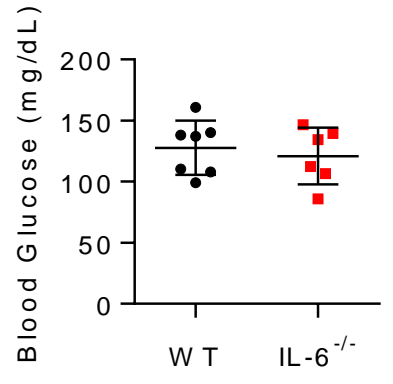

Figure S3 (Related to Figures 1,2,3 and 5). IL-6 knockout mice display hepatic steatosis, but not obesity

(A) Representative photographs of WT,sgp130Fc, and IL-6-/ mice at the age of 12 months.

(B) Body weight at 8 months of age in WT and IL-6-/ littermates $(n=14)$.

(C) Daily food-intake at 6 months of age $(n=9-12)$. Data represent mean food intake per mouse/cage per day averaged over a 2 week period.

(D) Fasting glucose levels at 6 months of age in WT and IL-6-/ littermates, $(n=6-7)$.

(E) Representative microphotographs showing steatosis by oil red O (ORO) staining in liver sections from WT and IL-6- ${ }^{-/}$littermates at 8 months of age and Image $\mathrm{J}$ processed image (Inset). Scale bars represent $20 \mu \mathrm{m}$. Quantification of ORO-stained area by ImageJ analysis (right) (n=6-7).

Data are represented as mean \pm SD. ${ }^{*} P<0.05$ by two-tailed, Student's $t$ test. 


\section{Figure S5}

A
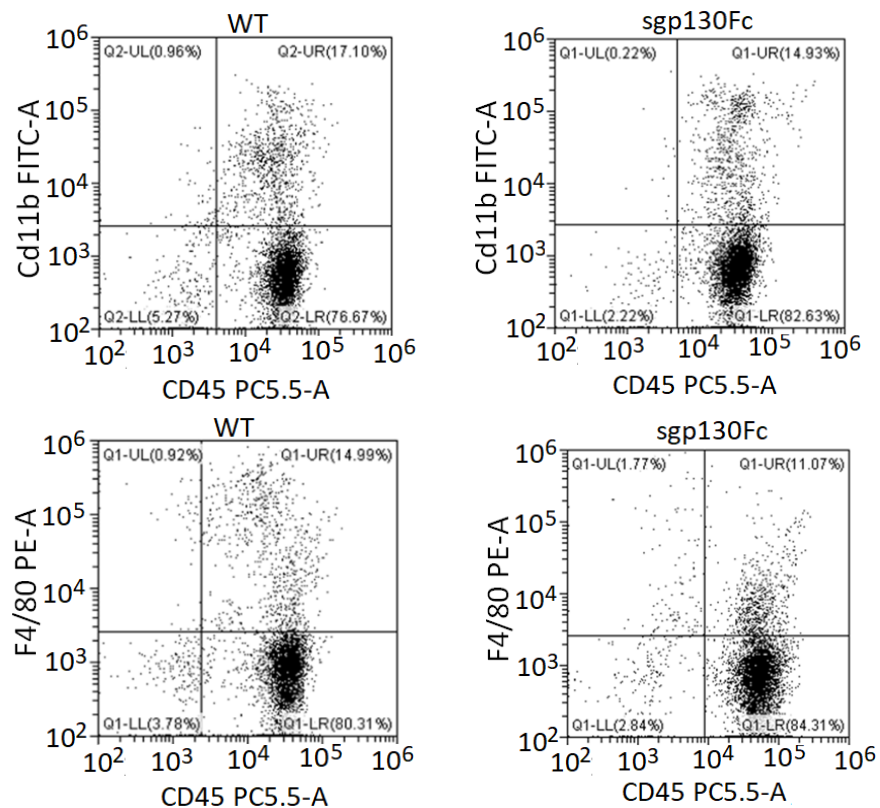

B
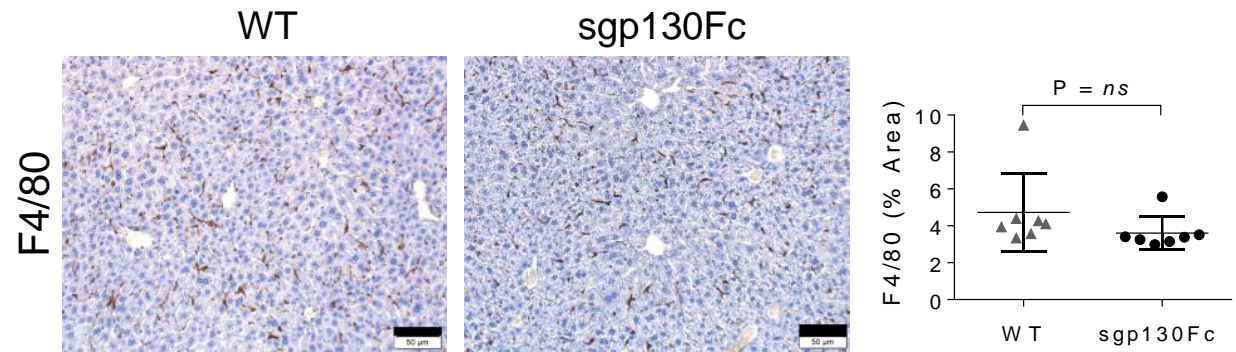

C
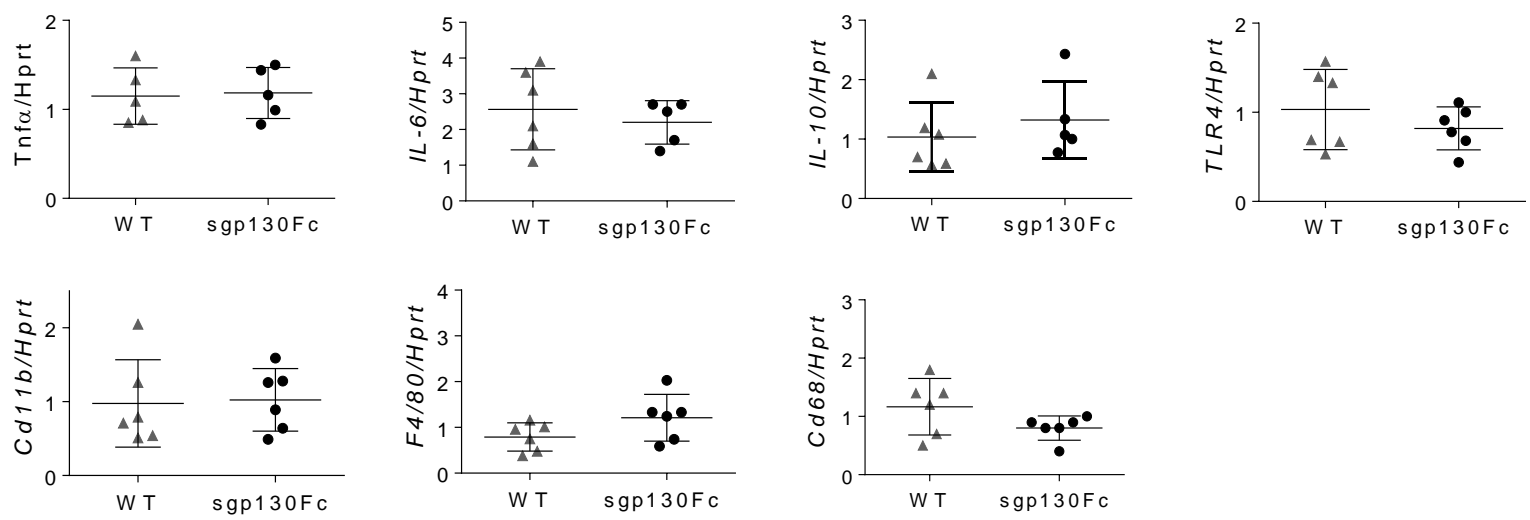

Figure S5 (Related to Figures 5). Inhibition of trans-signaling induces steatosis without hepatic inflammation.

(A) Representative plots from flow cytometry of double-positive Cd45+/Cd11b+and Cd45+/F4/80+ cells (upper right quandrants) in livers of sgp130Fc and WT littermates at 11 months of age.

(B) Representative photomicrographs of F4/80 immunostained paraffin embedded liver sections from 5-month old sgp130Fc and WT littermates and quantification (right) of F4/80+ cells as a function of area stained by ImageJ analysis, $(\mathrm{n}=8)$. Scale bars, $50 \mu \mathrm{m}$.

(C) Hepatic mRNA expression levels by real time qPCR of indicated inflammatory and monocyte markers in sgp130Fc vs. WT littermates at the age of 14 months.

Data are represented as mean $\pm \mathrm{SD}$. ${ }^{*} \mathrm{P}<0.05$ by two-tailed, Student's $t$ test. 


\section{Figure S6}

A

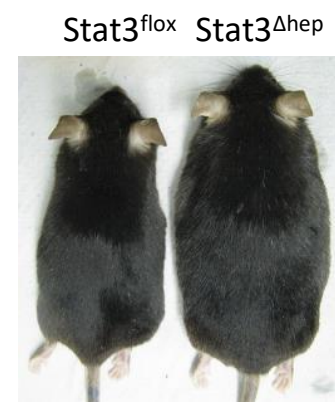

C

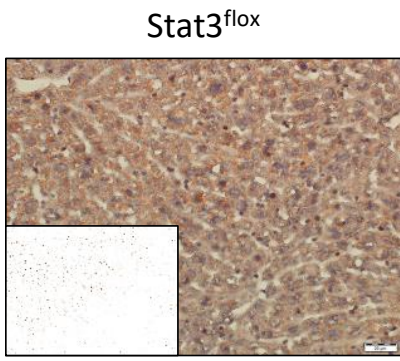

B
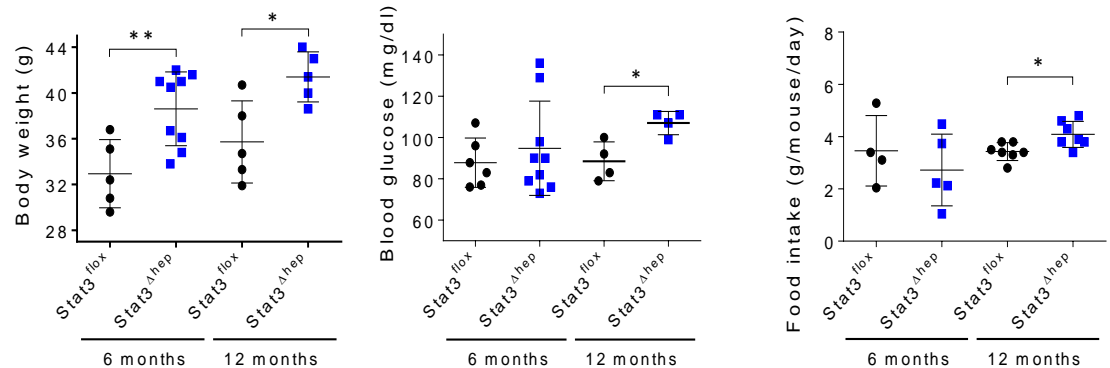

D
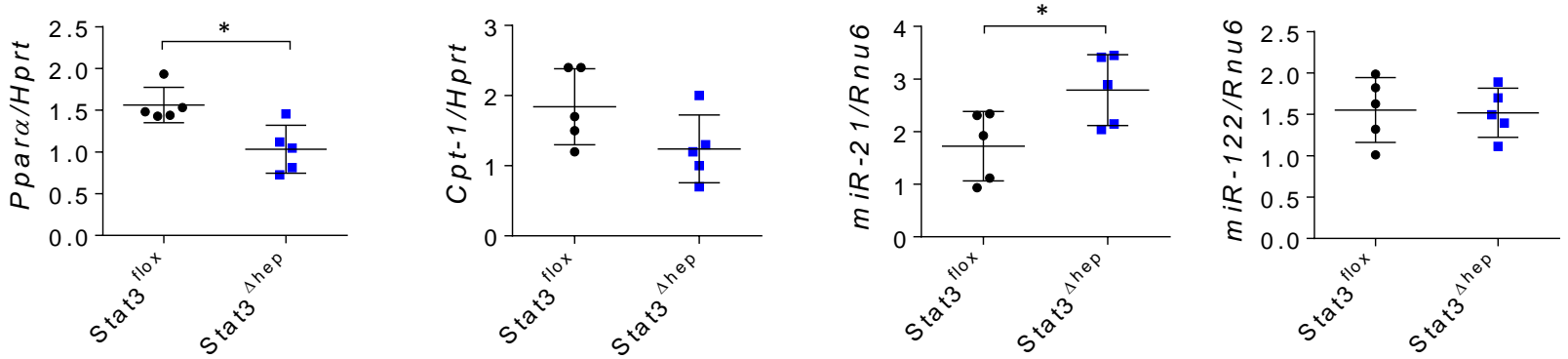

Figure S6 (Related to Figure 6). Trans-signaling protection against mature-onset metabolic syndrome may be mediated by Stat3 in hepatocytes.

(A) Photographic image showing representative Stat $3^{\Delta \text { hep }}$ and Stat $3^{\text {floxP }}$ mice at 12 months of age.

(B) Body weight, fasting blood glucose levels, daily food intake, and fasting-induced weight loss in 12 month old Stat $3^{\Delta \text { hep }}$ and Stat $3^{\text {floxP }}$ mice, $(n=5-8)$.

(C) Representative oil red O (ORO) stained liver sections from Stat3 ${ }^{\text {hhep }}$ and Stat3 $3^{\text {floxP }}$ mice at 12 months of age with ImageJ processed image (inset), and quantification of ORO-stained areas by ImageJ (right), $(n=4-5)$. Scale bars, $20 \mu \mathrm{m}$.

(D) Real time qPCR quantification of mRNAs encoding PPAR a, Cpt-1, and miR-21, and miR-122 in livers of Stat $3 \Delta$ hep and Stat3floxP mice at 12 months of age $(n=4-5)$.

Data are represented as mean $\pm S D$. ${ }^{*} P<0.05$, and ${ }^{* *} P<0.01$ by two-tailed, Student's t test. 


\section{Figure S7}
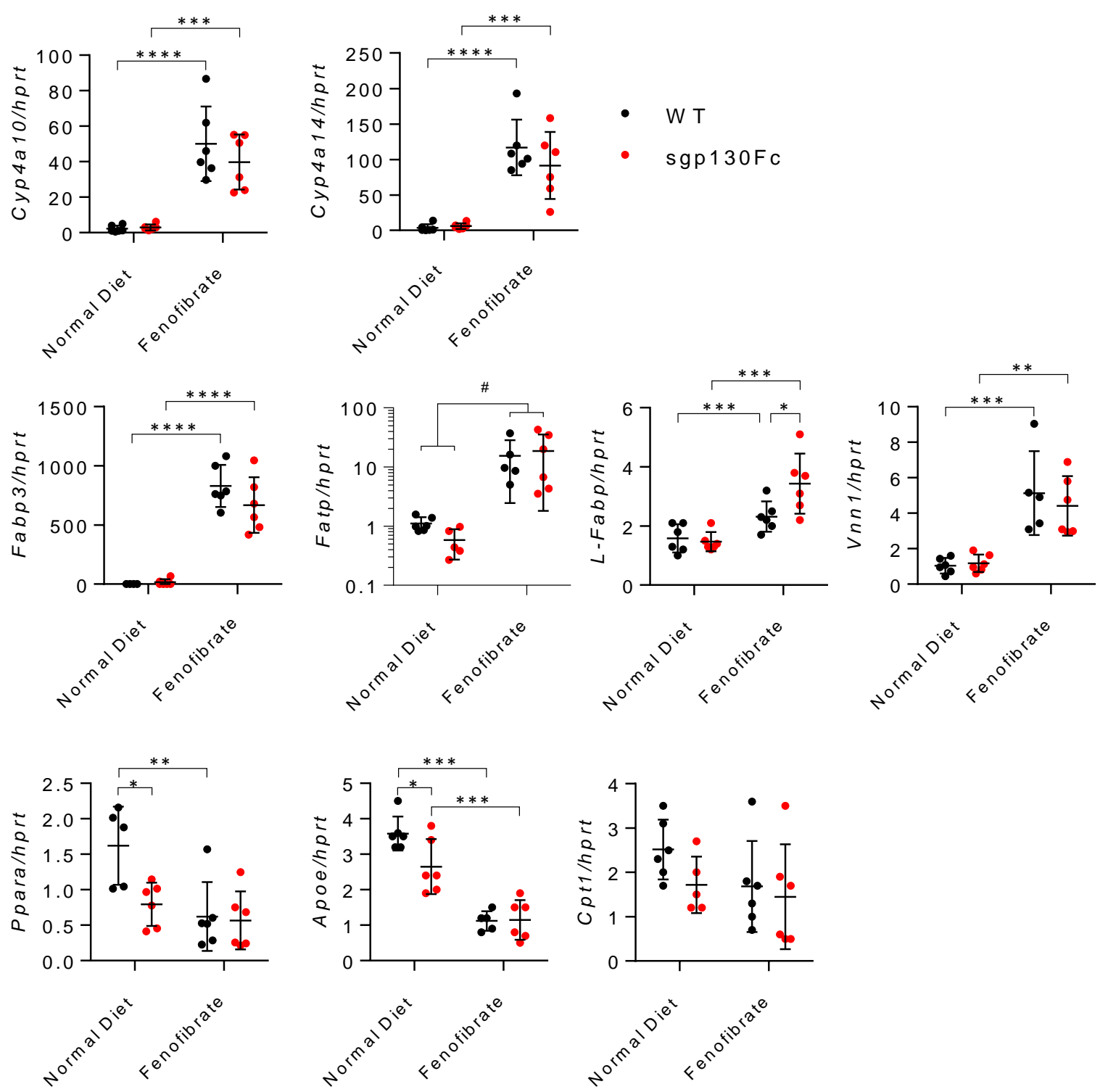

Figure S7 (Related to Figure 6). The effect of a fenofibrate on PPAR $\alpha$ and downstream target gene expression in the liver.

Sgp130Fc and wild type (WT) littermates aged 4.5 months were administered a Normal Chow Diet (NCD), or a NCD supplemented with Fenofibrate (FF) for 2.5 months. Data show hepatic mRNA levels of PPAR $\alpha$ and down-stream PPAR $\alpha$ target genes, Apoe, Cpt1, Cyp4a10, Cyp4a14, Fabp3, Fatp, L-Fabp and Vnn1 by real time $\mathrm{qPCR}$ analysis, $(\mathrm{n}=6)$.

Data are represented as mean $\pm \mathrm{SD} .{ }^{*} P<0.05,{ }^{* *} P<0.01,{ }^{* * *} P<0.001$ by two-way ANOVA. \# denotes main effect $(P<0.01)$ for diet by two-way ANOVA. Significant interaction between genotype and diet were reported for Fatp $(P=0.028)$. 


\section{Figure S8}

A

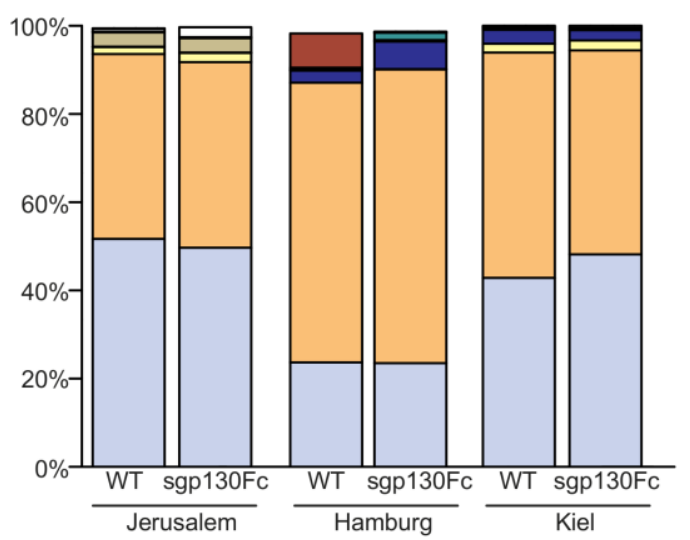

\begin{tabular}{l}
\multicolumn{1}{c}{ Phylum } \\
$\square$ Firmicutes \\
$\square$ Bacteroidetes \\
$\square$ Proteobacteria \\
$\square$ Bacteria unclassified \\
$\square$ Actinobacteria \\
$\square$ Tenericutes \\
$\square$ Verrucomicrobia \\
$\square$ Deferribacteres \\
TM7
\end{tabular}

B

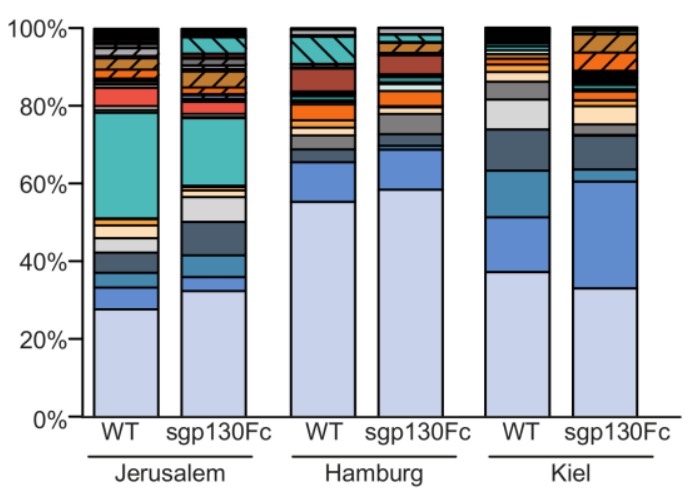

Genus

S24-7 unclassified

$\square$ Lachnospiraceae unclassified $\square$ Bacteroides $\square$ Lactobacillus $\square$ Prevotella $\square$ Bacteria unclassified $\square$ Oscillospira $\square$ Ruminococcus $\square$ Clostridium $\square$ Clostridiales unclassified ZuOdoribacter

Z7Rickenellaceae unclassified Z7B Bifidobacterium

Z7Bacteroidales unclassified IIJAckermansia

C

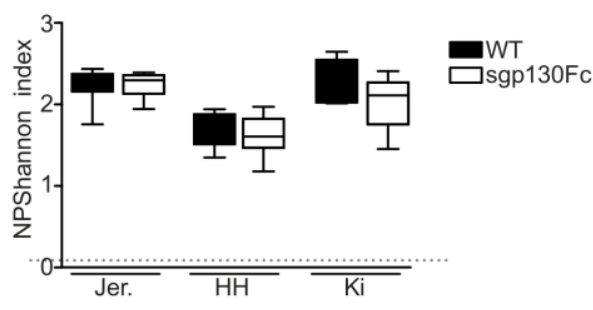

D
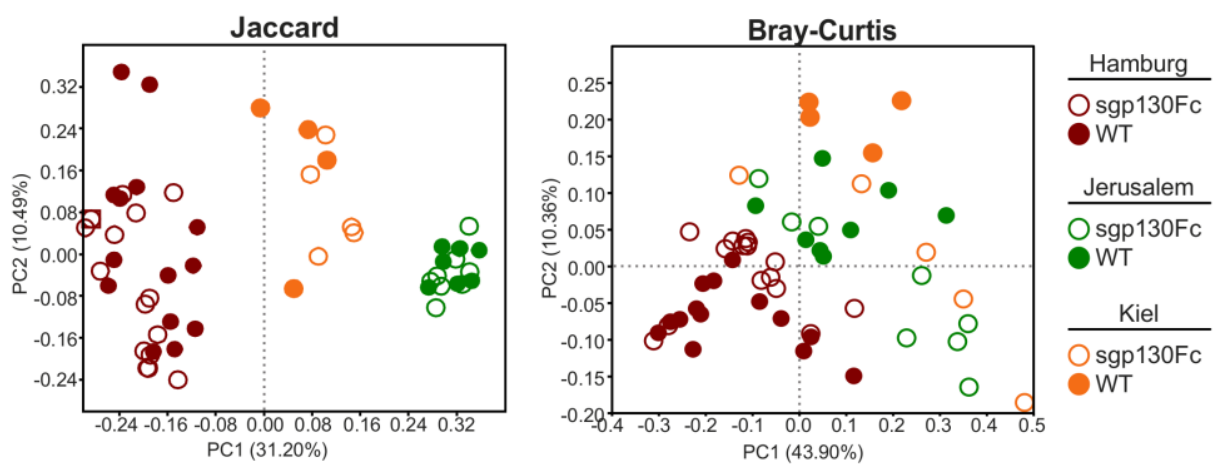

Figure S5. Inhibition of IL-6 trans-signalling does not alter gut microbiota composition. (A) Gut microbiota composition at the phylum level based on 16S rRNA gene sequencing in feces. (B) Relative bacterial abundance at genus level does not show alterations that can be associated with the genotype in three independent animal facilities. (C) Box plots of the Shannon diversity Indices show that total gut microbial diversity is independent of mouse genotype in three independent animal facilities. (D) left panel: Principal coordinate analysis ( $\mathrm{PCo}$ ) on Jaccard distance matrices demonstrates alterations in microbiota composition between different animal facilities. right panel: PCoA based on Bray-Curtis dissimilarity index of bacterial genus abundances. 
Table SI: $P$-values are shown for permutational analysis of variance (PERMANOVA) with Bonferroni correction for multiple testing

\begin{tabular}{|l|l|l|l|l|l|l|}
\hline & \multicolumn{3}{|c|}{ Jaccard } & \multicolumn{3}{c|}{ Bray-Curtis } \\
\hline facility & Jerusalem & Hamburg & Kiel & Jerusalem & Hamburg & Kiel \\
\hline Jerusalem & & 0.0003 & 0.0003 & & 0.0003 & 0.0942 \\
\hline Hamburg & 0.0003 & & 0.0003 & 0.0003 & & 0.0003 \\
\hline Kiel & 0.0003 & 0.0003 & & 0.0942 & 0.0003 & \\
\hline
\end{tabular}

